\title{
Synthesis,Mechanisms and Different Applications of Mesoporous Materials Based on Silica and Alumina
}

\author{
Heba M. Gobara \\ Egyptian Petroleum Research Institute, 1 Ahmed El-Zomor St., \\ Nasr City, 11727, Cairo, Egypt.
}

\begin{abstract}
SILICATE mesoporous materials have received widespread interest because of their potential applications. These novel materials, prepared either by soft- or hard-templating syntheses, become more and more important in many fields of science and technology such as adsorption, catalysis, separations, environmental processes, nanotechnology and biotechnology. According to IUPAC definition inorganic solids that contain pores with diameter in the size range of $2-50 \mathrm{~nm}$ are considered mesoporous materials, They also possess extremely high surface areas $\left(>700 \mathrm{~m}^{2} \mathrm{~g}^{-1}\right)$ and narrow pore size distributions. This article review shows the current state of art and outlines the recent patents in mesoporous materials research in three general areas: synthesis, various mechanisms involved for porous structure formation and applications of mesoporous materials based on silica and alumina.
\end{abstract}

Keywords: Mesoporous, Silica, Alumina, SBA-n, Synthesis mechanism and Application.

The Discovery of mesoporous materials

The first synthesis of M41S-type mesoporous silica was reported in 1992 by researchers in the Mobil Oil Corporation who discovered the remarkable features of this novel type of material and opened up a whole new field of research ${ }^{(1)}$. Since then, such highly ordered mesoporous silica materials have been intensively studied with regard to synthetic methods, mesostructure, surface modification, and applications as functional materials and in catalysis. In recent years, in addition to M41S molecular sieves, a variety of highly ordered mesoporous silica materials such as SBA, MSU, FDU, JLU, FSM, KIT have also been successfully synthesized using anionic, neutral, or nonionic surfactants, and the various assembly routes have been modified in order to optimize the synthesis process ${ }^{(2-9)}$.

According to the IUPAC definition, solids that contain pores with pore diameter (i) $>50 \mathrm{~nm}$ are called as macroporous, (ii) between 2-50 nm are mesoporous and (iii) $<2 \mathrm{~nm}$ are microporous. Some of the examples of different porous materials are listed in the Table 1 . Since the discovery of MCM-41 in

Corresponding author; email: hebagobara@ymail.com ,

Tel: +2 (02) 01223898196, +2(02) 01091446345,

Fax: +2 (02)227727433. 
1992 by Mobil scientists, mesoporous molecular sieves have attracted much interest because of their potential application in reactions or separations involving bulky molecules. The crucial problem is that MCM-41 has poor hydrothermal stability, either in stream or in hot water. This becomes a serious limitation to the application of this new material.

TABLE 1. Different types of porous materials.

\begin{tabular}{|c|c|c|c|}
\hline Type of Material & Pore Size & Examples & Pore Size Range $(\AA)$ \\
\hline \hline Macroporous & $>500 \AA$ & Porous glasses & $>500$ \\
\hline Mesoporous & \multirow{2}{*}{$20-500 \AA$} & Pillared layered clays & 10,100 \\
& & M41S & $16-100$ \\
& & SBA-15 & $80-100$ \\
& & SBA-16 & 50 \\
& & Diatom biosilica & $20-500$ \\
& \multirow{2}{*}{ Microporous } & Mesoporous alumina & 20 \\
& & Zeolites & $<14.2$ \\
& & Activated carbon & 6 \\
& & ZSM-5 & $4.5-6$ \\
& & Zeolite A & $3-4.5$ \\
& & Beta and Mordernite-Zeolites & $6-8$ \\
& & Faujasite & 7.4 \\
& & Cloverite & $6-1.32$ \\
\hline
\end{tabular}

Zhao et al. ${ }^{(10)}$ reported the synthesis of a novel mesoporous silica SBA-15 using an organic copolymer to organize the structure of a polymeric silica precursor template. It has a high surface area $\left(600-1000 \mathrm{~m}^{2} \mathrm{~g}^{-1}\right)$, thick walls, and uniform tubular channels with tunable pore diameters in the range of 5-30 nm, significantly larger and with higher hydrothermal stability than those of MCM-41. Thus, it has attracted great attention in the field of catalysis, separation, sensor design and nanoscience.

\section{Ordered mesoporous materials}

The pore size is one of the most important physical parameters of ordered mesoporous materials (OMMs) for practical applications, and must be designed depending on their application. Generally, tailoring the pore sizes of OMMs can be achieved either by using surfactants with various chain lengths, or by adding swelling agents, such as 1, 3, 5-trimethylbenzene (TMB), amines, and decane and/or hydrothermal treating under different temperatures. Very recently, Chang and co-workers ${ }^{(11)}$ used a blend of cationic-cationic surfactants, and Ryan and co-workers ${ }^{(12)}$ used the mixed triblock copolymer surfactants to tailor the pore morphology. Lee et al. ${ }^{(13)}$ utilized the hexadecyl-ether-type surfactant and CTAB as co-surfactant to tailor the pore size of OMMs. However, the surfactants with various chain lengths are not always available and the enlargement of pore size by adding swelling agents would very easily result in the loss of the structural periodicity and poor reproducibility.

Egypt. J. Chem. 59, No. 2 (2016) 
One of the most important studied systems has been SBA-n type mesoporous silica, synthesized by condensation of alkoxysilanes such as tetraethoxysilane (TEOS) in the presence of commercially available nonionic poly (EOn-POmEOn) triblock copolymers as a template as shown in Fig. 1. These materials have controllable pore size (ranging from 2 to $30 \mathrm{~nm}$ ) and thick pore walls (ranging from 1.5 to $8.0 \mathrm{~nm}$ ), as well as much higher hydrothermal stability than M41Stype materials.

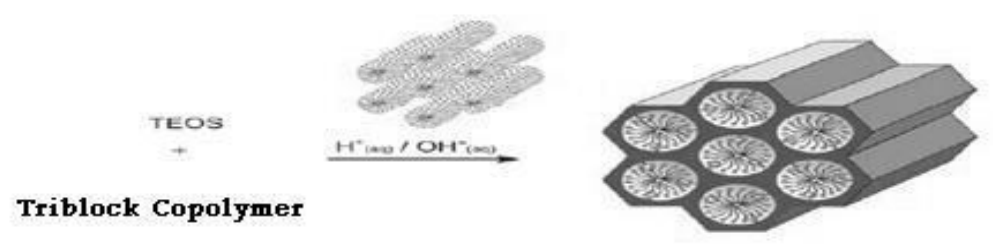

Fig. 1. Co-condensation method (direct synthesis) for SBAn formation.

Jin et al. ${ }^{(14)}$ reported a two-step method for synthesis of well-ordered SBA15-type and SBA-16-type mesoporous silica materials under mildly acidic conditions by separating the hydrolysis and the condensation steps of the silica source in a controlled $\mathrm{pH}$ range higher than the isoelectric point of silica $(\mathrm{pH} 2-$ 5). It is known that the fluoride ion can serve as a powerful promoter for hydrolytic condensation of alkoxysilanes, such as TEOS, in aqueous media containing amphiphilic surfactants and thus give thermally and hydrothermally stable mesoporous silica. Voegtlin et al. ${ }^{(15)}$ have described the synthesis of mesoporous silica in neutral to basic media by using a nonionic triblock copolymer (Pluronic P123) as template with the addition of fluoride whilst Schmidt-Winkel et al. (16) have reported the synthesis of SBA-15-type mesoporous silica rods with uniform channels at moderate acidity $(\mathrm{pH}<2.7)$, without compromising the long-range symmetry as shown in Fig. 2.

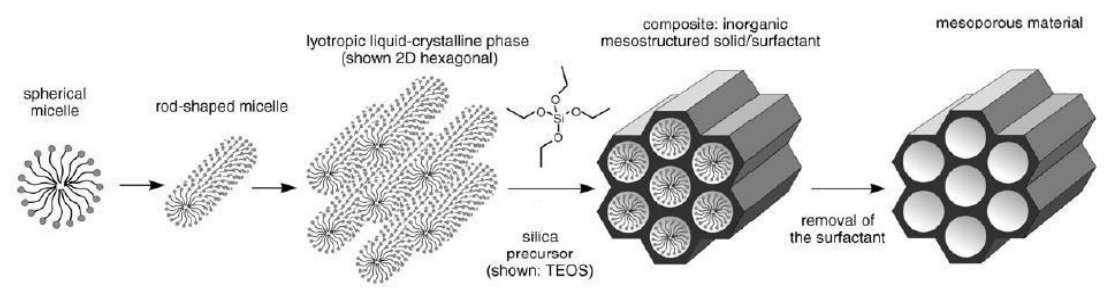

Fig. 2. Formation of mesoporous materials by structure-directing agents.

\section{Transition metal incorporation}

The incorporation of transition metal ions in these mesoporous structures is a very useful method for providing this material with potential catalytic applications. Tungsten is one of the most-studied transition metal ions (along with as $\mathrm{Al}, \mathrm{Ti}, \mathrm{V}$ and $\mathrm{Sn}$ ) incorporated into MCM 41, MCM-48 and SBA-15 as 
listed in Table 2, due to its interesting catalytic properties in different reactions. Tungsten is widely used for the metathesis and isomerization of alkenes, selective oxidation of unsaturated compound, dehydrogenation of alcohols and hydrodesulfurization and hydrocracking of heavy fractions in the petroleum chemistry, the subject most heavily researched. In addition, research groups have attempted to synthesize mesoporous tungsten oxide materials or incorporate tungsten into nonsiliceous mesostructured materials $\left(\mathrm{TiO}_{2}\right)$ and siliceous mesoporous molecular sieves (e.g., silica, M41S, SBA-n) by impregnation, cogelation, insertion, or grafting ${ }^{(17)}$. In these materials, the structure of the nonsupported tungsten oxide mesoporous materials is not stable and will collapse on calcination. Thus, incorporating tungsten into nonsiliceous or siliceous mesoporous materials has attracted considerable interest because of their diverse compositions that lead to potential applications in catalysis.

TABLE 2. Metal loaded mesoporous silica and alumina.

\begin{tabular}{|c|c|}
\hline $\begin{array}{l}\text { Type of Porous } \\
\text { Materials }\end{array}$ & $\begin{array}{l}\text { Type of Metal } \\
\text { incorporated }\end{array}$ \\
\hline MCM-41 & $\mathrm{Al}$ \\
\hline MCM-41 & $\mathrm{Ti}$ \\
\hline MCM-41 & $\mathrm{V}$ \\
\hline MCM-41 & $\mathrm{B}$ \\
\hline MCM-41 & $\mathrm{Mn}$ \\
\hline MCM-41 & $\mathrm{Sn}$ \\
\hline MCM-41 & $\mathrm{Ga}$ \\
\hline MCM-41 & $\mathrm{Fe}$ \\
\hline MCM-41 & $\mathrm{Zr}$ \\
\hline MCM-41 & $\mathrm{Cr}$ \\
\hline MCM-41 & Mo \\
\hline MCM-41 & $\mathrm{Nb}$ \\
\hline MCM-48 & $\mathrm{Fe}$ \\
\hline MCM-48 & $\mathrm{Ti}$ \\
\hline MCM-48 & $\mathrm{Cr}$ \\
\hline MCM-48 & $\mathrm{V}$ \\
\hline MCM-48 & $\mathrm{Mn}$ \\
\hline SBA-15 & $\mathrm{Zn}$ \\
\hline SBA-15 & $\mathrm{Eu}$ \\
\hline SBA-15 & $\mathrm{Ti}$ \\
\hline SBA-15 & $\mathrm{Sn}$ \\
\hline SBA-15 & $\mathrm{Ni}$ \\
\hline SBA-15 & $\mathrm{Fe}$ \\
\hline SBA-15 & $\mathrm{Pd}$ \\
\hline
\end{tabular}

The incorporation of aluminum into SBA-15 by post synthetic and direct Egypt. J. Chem. 59, No. 2 (2016) 
methods has been reported ${ }^{(18)}$. During materials preparation via post synthetic methods often metal oxides are formed in the channels or on the external surface. Metal oxides formed in the mesopores will block the pores partially or fully, thereby reducing surface area, pore volume, and pore diameter, or play a negative role in catalysis Yue et al. ${ }^{(19)}$ reported the direct synthesis of AlSBA-15 and found that catalytic activity of AlSBA-15 in cumene cracking is higher as compared to AlMCM-41. However, the highly acidic synthesis gel required for the formation of SBA-15 limits the direct incorporation of high amounts of trivalent metal ions into the neutral silica framework and requires a post synthetic treatment to remove octahedral aluminum. A different strategy is the transformation of amorphous SBA-15 walls into crystalline aluminosilicates. It has been found for AlMCM-41 that the extent of Al incorporation and substitution in the tetrahedral framework and the catalytic activity are greatly dependent on the Al source which is used for the preparation of these materials. Janicke $e a l^{(20)}$ and Reddy and Song ${ }^{(21)}$ have reported that $\mathrm{Al}$ isopropoxide is a better source for the incorporation of Al (III) in MCM-41. Recently, Hartmann et al. ${ }^{(22)}$ have reported the effect of $\mathrm{Al}$ source on the incorporation of MCM-48 and found that the maximum incorporation of $\mathrm{Al}$ and large pore diameter on the MCM-48 were achieved by using $\mathrm{Al}$ isopropoxide as the $\mathrm{Al}$ source. Materials with uniform and tunable pore sizes are expected to play an important role in a number of applications that range from catalysis, to molecular separations and sorption of very bulky molecules, and to the fabrication of semiconductors, semiconductor nanowires and low dielectric devices.

\section{Synthesis of mesoporous silicate}

\section{Hydrothermal method}

Mesoporous silicates are generally prepared under "hydrothermal" conditions. The typical sol-gel process is involved in the "hydrothermal" process. A general procedure includes several steps. First, a homogeneous solution is obtained by dissolving the surfactant(s) in a solvent. Water is the most common solvent and medium. Silicate precursors are then added into the solution where they undergo hydrolysis catalyzed by an acid or base catalyst and transform to a sol of silicate oligomers. As a result of the interaction between oligomers and surfactant micelles, cooperative assembly and aggregation give precipitation from a gel. During this step, microphase separation and continuous condensation of silicate oligomers occur. The formation of mesoporous silicates is rapid, only 3-5 min in cationic surfactant solutions, which is reflected by the precipitation.

Many attempts have been conducted to investigate the mesostructure transition by using an in situ X-ray diffraction (XRD) technique ${ }^{(18,23)}$. The results reveal that MCM-41 products are disordered before the formation of gels. Once precipitation is produced, well-ordered mesostructures can be detected. The formation of mesostructures is slow if nonionic surfactants are used as templates, normally in $30 \mathrm{~min}$ or even longer. Tetramethoxysilane (TMOS) as an inorganic silicate precursor results in a faster formation of mesoporous silica structures than TEOS. In comparison with them, tetrabutoxysilane (TBOS) slows down the synthesis. This phenomenon can be attributed to their hydrolysis rates. 
Subsequent solidification and reorganization further proceed to form an ordered mesostructure.

Hydrothermal treatment is carried out to induce the complete condensation and solidification and improve the organization. The resultant product is cooled down to room temperature, filtered, washed, and dried. Mesoporous material is finally obtained after the removal of organic template(s). Ordered mesoporous silicates are generally synthesized under basic or acidic conditions. Neutral solutions are unsuitable to get ordered silicate mesostructures, because of too rapid polymerization and cross-linking rates of silicates at $\mathrm{pH}$ of $6.0-8.5$ to control the surfactant-templating assembly. The addition of fluoride catalysts can lead to ordered solids at a wide range of $\mathrm{pH}$ values even including the neutral conditions owing to the well-controlled rates of hydrolysis and condensation of silica precursors.

\section{(i)Basic synthesis}

Under basic conditions, in the $\mathrm{pH}$ range from 9.5 to 12.5 , the polymerization and cross-linkage of silicate species are reversible. Therefore, silicate precursors that can be used to prepare ordered mesoporous silicas are diverse, for example, silica gels, colloidal sols, water glass (sodium silicates), silica aerogels, or TEOS. Except TEOS, the others have high polymerization degrees of silicates. Hydrothermal treatment is necessary to prepare ordered mesoporous silicates when they are used as silicate precursors. Mixed silicate precursors were used in the synthesis of MCM-41 by Mobil scientists, perhaps on the consideration that the pore walls of MCM-41 are amorphous ${ }^{(24)}$. It is found that TEOS is the most convenient and efficient silicate precursor in the laboratory ${ }^{(25)}$. Sodium hydroxide, potassium hydroxide, $\mathrm{NH}_{3} \cdot \mathrm{H}_{2} \mathrm{O}$, tetramethyl -ammonium hydroxide (TMAOH, $\left.\left(\mathrm{CH}_{3}\right)_{4} \mathrm{NOH}\right)$ and tetraethylammonium hydroxide (TEAOH, (C2H5)4NOH) can be used as a base.

The latter two quaternary ammonium bases are expensive and are mostly used in the syntheses of hydrogen-type mesoporous aluminosilicates. Owing to the weak alkalinity of $\mathrm{NH} 3 . \mathrm{H} 2 \mathrm{O}$, disordered mesoporous silica materials are sometimes obtained with it. The $\mathrm{pH}$ value of media varies during the synthesis time. It is distinctly reduced at the beginning caused by the hydrolysis of silicates and then slightly increased due to the crosslinkage of silica species. Therefore, high-quality MCM-41 can be synthesized in the pH range between 11.0 and 11.5 adjusted by adding sulfuric or acetic acid during the synthesis.

\section{(ii) Acidic synthesis}

In 1994, a breakthrough for the synthesis of highly ordered mesoporous silicates under strongly acidic conditions was realized by Huo et al. ${ }^{(25)}$. Mesoporous silicas SBA-1 were prepared by using cetyltriethyl ammonium bromide $\left(\mathrm{C}_{16} \mathrm{H}_{33} \mathrm{~N}\left(\mathrm{CH}_{2} \mathrm{CH}_{3}\right)_{3} \mathrm{Br}, \mathrm{C}_{16} \mathrm{TEABr}\right)$ as a template and TEOS as a silica source.

Thereafter, SBA-15 and SBA-16 large-pore mesoporous silicas were 
synthesized by using triblock copolymers as templates under acidic conditions. The characteristics for an acidic synthesis are as follows:

\section{(1) $\mathrm{pH}$ dependence}

The synthesis of mesoporous silica is accelerated by lowering the $\mathrm{pH}$ values of the solution. A high acid concentration leads to a fast precipitation rate. On the other hand, acid catalyst at low concentration favors a slow condensation rate of silicate species. When strong acid $\mathrm{HCl}$ serves as a catalyst, the optimum $\mathrm{pH}$ value is below 1 . The higher the concentration of $\mathrm{H}^{+}$is, generally the more hydrophilic the block copolymer is. This is because the PEO moieties of block copolymers are readily protonized in the strongly acidic media. Highly concentrated $\mathrm{HCl}(>4 \mathrm{M})$ is, however, not recommended, because low-quality products are often obtained. In contrast, using low acidic concentration $(0.5 \mathrm{M}$ $\mathrm{HCl})$ and n-butanol additive slows the condensation rate of the inorganics and can easily yield a highly ordered 3D cubic SBA-16 mesostructure ${ }^{(26)}$. Besides $\mathrm{HCl}$, strong acids such as $\mathrm{HNO}_{3}, \mathrm{HBr}, \mathrm{HI}$, and $\mathrm{H}_{2} \mathrm{SO}_{4}$ can be used as catalysts.

Seldom cases adopt weak acids, like $\mathrm{H}_{3} \mathrm{PO}_{4}$ and acetic acid (HAc), due to the low-quality products. It is unknown whether the products contain phosphor in $\mathrm{H}_{3} \mathrm{PO}_{4}$-containing systems. The precipitation of mesoporous silicates is extremely slow in the $\mathrm{pH}$ value range from 1 to 2 , probably because this is around the isoelectric point of silica. On account of this character, Prouzet and co-workers designed a method to investigate the formation mechanism of mesostructures template by nonionic surfactants. The stable prehydrolyzedsilicate sols in the solutions at $\mathrm{pH}$ make the detailed analysis possible. The main components, soluble silicate oligomers and nonionic surfactants micelles, do show interactions at this stage.

\section{(2) Controllable morphology}

The acidic synthesis is suitable for the formation of mesoporous silicates with diverse morphologies, such as single crystals, thin films, fibers, spheres, etc. It may be related to the sol-gel chemistry of silicates. Linear silicate oligomers are the main products from the hydrolysis of silicates under acidic conditions that favor various regular morphologies. Base catalysis leads to a fast polymerization and condensation of silicates, yielding 3D silicate networks. The morphology is sometimes difficult to control. Spherical particles are the most common product.

\section{(3) Irreversible reaction}

The irreversible polymerization of silicate species will lead to failure of the synthesis once the gel forms. In contrast, the hydrolysis of silicates is reversible under basic conditions. Ordered mesostructures can be synthesized even if a gel appears.

\section{(4) Simple silica source}

Siliceous oligomers and monomers are suitable precursors owing to the irreversible polymerization of silicates under acidic conditions. TEOS is the optimal choice. Sodium metasilicate $\left(\mathrm{Na}_{2} \mathrm{SiO}_{3}\right)$ that can generate small siliceous 
oligomers in a fast acidification process can also be used as a precursor. The simultaneously generated sodium salts facilitate the formation of mesostructures.

\section{(5) Low processing temperature}

The synthesis of mesoporous silica (SBA-3) is carried out at room temperature by using cationic surfactant as a SDA. Heating or hydrothermal treatment is not adopted.

(6) Only few examples for phase transformation

A surfactant generally templates one mesostructure; for example, CTAB yields the $2 \mathrm{D}$ hexagonal mesostructure $\mathrm{SBA}-3$ and $\mathrm{C}_{16} \mathrm{TEABr}$ gives the $3 \mathrm{D}$ simple cubic mesostructure SBA-1. Compared with basic synthesis, it is much more difficult to change the mesostructure by simply adjusting concentration, temperature, etc. Only few examples for transformations are observed. For example, in the synthesis of 3D SBA-12 with mixed hexagonal close packing (hcp) and cubic close packing (ccp) phases, a hydrothermal treatment at $100{ }^{\circ} \mathrm{C}$ for 3 days can transform it to the 2D hexagonal mesostructure.

(7)There is no need for a washing step

(8)The addition of inorganic salts like $\mathrm{KCl}, \mathrm{NaCl}$.

$\mathrm{Na}_{2} \mathrm{SO}_{4}$ and $\mathrm{K}_{2} \mathrm{SO}_{4}$ can accelerate and improve the synthesis while organic solvent additives may reduce the formation rate in acidic nonionic surfactant systems. With the addition of inorganic salts, high-quality SBA-15 can be synthesized even at low temperature $\left(-10{ }^{\circ} \mathrm{C}\right)$ and low tri-block copolymer P123 concentration.

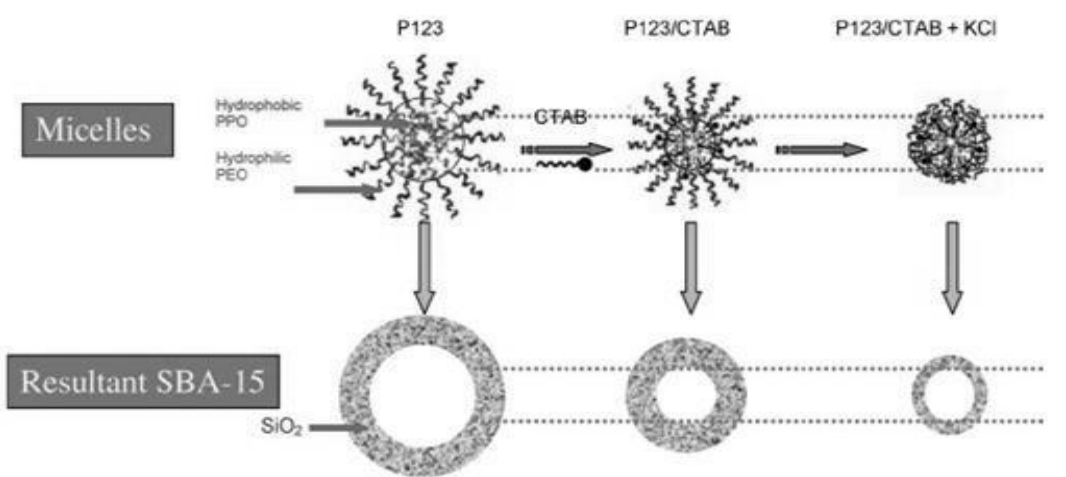

Fig. 3. The effect of $\mathrm{CTAB}$ and $\mathrm{KCl}$ on the micelles of $\mathrm{EO}_{20} \mathrm{PO}_{70} \mathrm{EO}_{20}$ and the resultant mesoporous silicas.

(iii) Synthesis (reaction) temperature

In the solution synthesis, the reaction temperature is relatively low, ranging from -10 to $130{ }^{\circ} \mathrm{C}$. The most convenient temperature is room temperature. Two factors can be referred to, CMT and cloud-point (CP), to select the temperature. The temperature is normally higher than the CMT values of the surfactants. The CMT values are relatively low for cationic surfactants. In the cationic surfactant

Egypt. J. Chem. 59, No. 2 (2016) 
templating cases, the assembly rate of the templating surfactants slows down with the decrease of the synthetic temperature that facilitates the preparation of high quality mesoporous silicates. Heating is unnecessary and room-temperature synthesis is feasible. When nonionic surfactants are used as templates, the reaction temperature is normally higher than room temperature because of their higher CMT values. But also a low-temperature strategy has been developed to synthesize highly ordered mesoporous silicates FDU-12 with exceptionally large pore sizes.

A common idea is to decrease the synthetic temperature, which reduces the reaction rate and thereby improves the crystalline regularity. In the synthesis of SBA-15 templated by triblock copolymer P123, the optimal synthetic temperature is $35-40^{\circ} \mathrm{C}$, due to the solubility limit and the CMT value for the formation of micelles. The reaction temperature is high when using block copolymers with high CMT and CP values. It is found that ordered mesoporous silicates can only be obtained at the temperature higher than $90^{\circ} \mathrm{C}$ in the triblock copolymer P85 $\left(\mathrm{EO}_{26} \mathrm{PO}_{39} \mathrm{EO}_{20}\right)$ and $\mathrm{P} 65\left(\mathrm{EO}_{20} \mathrm{PO}_{30} \mathrm{EO}_{20}\right)$ systems, both of which have a $\mathrm{CP}$ value of $82{ }^{\circ} \mathrm{C}$ in water. This phenomenon can be explained by the fact that the $\mathrm{CP}$ values of surfactants may be influenced by the solvents. In the presence of $2 \mathrm{M} \mathrm{HCl}$ and ethanol, the $\mathrm{CP}$ values of P85 and P65 are higher than $95^{\circ} \mathrm{C}$. Because the hydrolysis of TEOS generates ethanol, the reaction temperature in the acidic P85 and P65 systems is a little higher than their CP values in water.

The nature of the inorganic precursor is not a decisive factor that would restrict the synthetic temperature. However, high temperature is adopted in the cases of inorganic precursors with high polymerization degrees. When silica aerogel or water glass is used as silica precursor, a high temperature of $100^{\circ} \mathrm{C}$ favors the rehydrolysis, cross-linking, and "uniform distribution" of silicate species, the assembly of mesostructures, and the formation of ordered stable mesoporous silicates.

\section{(iv) Hydrothermal treatment}

Hydrothermal treatment is one of the most efficient methods to improve mesoscopic regularity of products. After the solution reaction, the porosity can only be obtained after the removal of templates from as-synthesized inorganicorganic composites. Different removal methods certainly influence the characteristics of mesoporous materials. The most common method to remove templates is calcinations owing to the easy operation and complete elimination. Organic surfactants can be totally decomposed or oxidized under oxygen or air atmosphere. This method is mostly applied into the cases of mesoporous silicates, aluminosilicates, metal oxides, and phosphates. The temperature programming rate should be low enough to prevent the structural collapse caused by local overheating. Two-step calcinations were adopted by Mobil's scientists, the first $1 \mathrm{hr}$ under nitrogen to decompose surfactants and the following $5 \mathrm{hr}$ in air or oxygen to burn them out. This complicated procedure was then simplified. The first calcination step under nitrogen can be substituted by heating in air with 
a low rate. Heating the as-synthesized SBA-15 materials with a rate of $1-2^{\circ} \mathrm{C} / \mathrm{min}$ to $550{ }^{\circ} \mathrm{C}$ and keeping this temperature for 4-6 hr can completely remove triblock copolymer templates. Calcination temperature should be lower than the stable temperature for mesoporous materials and higher than $350^{\circ} \mathrm{C}$ to totally remove PEO-PPO-PEO type surfactants or $550^{\circ} \mathrm{C}$ for long-chain alkyl surfactants. Higher calcination temperature would lead to lower surface areas, pore volumes, and surface hydroxyl groups and higher cross-linking degrees of mesoporous materials.

But these materials possess higher hydrothermal stability due to the higher cross-linking degrees. During the calcination, cationic cetyltrimethylammonium surfactants undergo several decomposition steps. The majority of the surfactants first break down to form hexadecane and a trimethylamine species in the temperature range of 100 to $220^{\circ} \mathrm{C}$. At the same time, a small quantity of surfactant molecules, which are more strongly bonded to the inorganic hosts, decomposes between 195 and $220^{\circ} \mathrm{C}$, via the mechanism analogous to the thermal decomposition of the pure surfactant under the same experimental conditions. Several decomposition residues remain within the inorganic hosts, and the surface is notably hydrophobic. Upon continuous heating at high temperatures (to $550^{\circ} \mathrm{C}$ ), the carbon chain rearrangements are eliminated and the surface available becomes hydrophilic. The drawbacks of calcination are the unrecovered of surfactants and the sacrifice of surface hydroxyl groups. Moreover, it is unsuitable for thermally unstable and air-sensitive materials, such as sulfides and organic frameworks.

Extraction is a mild and efficient method to remove surfactants and get porosities without distinct effects on frameworks. Ethanol or THF can be used as an organic extracting agent. A small amount of hydrochloric acid is added in the extracting agent to improve the cross-linkage of frameworks and to minimize the effects on mesostructures. More than 95\% triblock copolymer P123 from as synthesized SBA-15 can be extracted and recovered to reuse. SBA-15 materials templated by the recovered $\mathrm{P} 123$ have good quality, indicating a minor destruction of triblock copolymer surfactants during the solvent extraction. With the aid of sulfuric acid, triblock copolymers in SBA-15 and SBA-16 mesostructures can be removed tailored pore channels and structures can then be achieved.

\section{Nonaqueous synthesis}

Nonaqueous synthesis is a very convenient method to prepare ordered mesoporous materials especially for mesoporous thin films, membranes, monoliths, and spheres. This method has become more and more powerful. Most of the syntheses conducted in the nonaqueous media adopt the well known EISA process ${ }^{(27)}$. For the preparation of mesostructured silica films, TEOS is dissolved in the organic solvent (normally ethanol, THF and acetonitrile) and prehydrolyzed with stoichiometric quantities of water (catalyzed by acids, such as $\mathrm{HCl}$ ) at a temperature of $25-70{ }^{\circ} \mathrm{C}$. Then low polymerized silicate species can

Egypt. J. Chem. 59, No. 2 (2016) 
randomly assemble with surfactants. Upon solvent evaporation, the silicate species further polymerize and condense around the surfactants. The polymerization rate is gradually increased due to the increasing acid concentration during the solvent evaporation. Simultaneously, templating assembly in the concentrated surfactant solution occurs, resulting in the formation of ordered mesostructures. The process is very fast and needs only several seconds. Mostly solvents with weak polarity are used. Surfactants lose the hydrophilic/hydrophobic properties in the weak polarity solvents because both hydrophilic and hydrophobic segments can interact with these solvents. The surfactant self assembly would be inhibited. However, the assembly can be induced upon the solvent evaporation. Nonpolar and oily solvents are seldom adopted. In toluene or xylene solution, silica nanowires with adjustable diameters were synthesized with P123 and F127 $\left(\mathrm{EO}_{106} \mathrm{PO}_{70} \mathrm{EO}_{106}\right)$ by the EISA approach. The formation of this kind of arrays corresponds to the measurements reveal, however, large domain ordering. One can see ordered arrays everywhere. The lack of XRD diffraction peaks can be attributed to the extremely fast formation rate of the mesostructure that causes non-uniform micelles. The non uniformity is difficult to observe by TEM images due to the minor discrepancy. However, this is magnified by the powder X-ray diffraction. At the same time, the samples normally require certain substrates for controlled deposition, which imposes a strain field, generating a uniaxial lattice distortion. This distortion lowers the mesostructure symmetry that can be qualitatively and quantitatively analyzed.

\section{Post synthesis treatment}

(i) Secondary synthesis

To improve thermal stability of mesoporous silicates, secondary synthesis is generally used to increase the pore wall thickness or enhance the local ordering, which can be achieved by either grafting or hydrothermal treatment. After MCM41 materials are treated by $\mathrm{AlCl}_{3}$ vapor or react with $\mathrm{AlCl}_{3}$ solution, they exhibit better mechanical and hydrothermal stability than the parent materials. This is related to the increase of pore wall thickness and cross-linking degree and the reparation of defects in frameworks. Immersing mesoporous silica thin films in ethanol vapor before the air drying process promotes structural regularity. Post synthesis treatment by ammonia gas is beneficial for the thermal stability of mesoporous silicate thin films, perhaps due to the improvement of the crosslinkage of silica networks under alkaline conditions. The improvement of stability or the expansion of pores of mesoporous silica solids is distinct after the secondary hydrothermal treatment in the presence of ammonia or $\mathrm{N}, \mathrm{N}$ dimethyldecylamine aqueous solution. TEOS can also be utilized in the secondary synthesis. It should be noted that this kind of secondary synthesis is more effective for as-synthesized materials than calcined ones.

Xia and co-workers ${ }^{(28)}$ reported a method to restructure calcined mesoporous silica MCM-41 by utilizing it as the "silica source" in a synthesis gel consisting $\mathrm{CTAB}, \mathrm{TMAOH}$ and water. After the secondary hydrothermal treatment, MCM41 material exhibits extremely high hydrothermal stability, which is ascribed to the improvement of condensation degrees of silicates.

Egypt. J. Chem. 59, No.2 (2016) 


\section{(ii) Recrystallization}

Recrystallization is a very efficient method to improve the regularity of mesoporous materials. However, only a few research groups realize this method, which is easily confused with the hydrothermal treatment. In fact, both processes are largely different. Recrystallization is a procedure in which as-synthesized powder samples without washing are placed into deionized water at $100-150{ }^{\circ} \mathrm{C}$ for several days (sometimes even 1 week). The quality (ordering, thermal stability, etc.) can be improved for most materials, sometimes accompanied with the enlargement of pore sizes ${ }^{(25)}$. This process is quite complicated. Dissolution and crystallization of silicate species and reorganization of mesostructures may take place. In comparison with the hydrothermal treatment, the reorganization rate in this process may be slower and more localized by reason of separated surfactants and un-reacted silicate species. For the recrystallization, unwashed samples are favorable, because residues of acid or base catalysts, silicate oligomers, and surfactants would facilitate the reorganization of mesostructures. Huo et al. ${ }^{(25)}$ recrystallized mesoporous silica MCM-41 from a basic CTAB surfactant system and found that it had more than seven XRD diffraction peaks. If mesoporous silica thick membranes templated by P123 from the EISA approach are recrystallized at $100{ }^{\circ} \mathrm{C}$ for 3 days, the mesostructure regularity is much improved as evidenced by at least three well-resolved XRD peaks. The resultant product has a highly ordered 2D hexagonal mesostructure with much larger surface area $\left(840 \mathrm{~m}^{2} / \mathrm{g}\right)$, pore size $(9.0 \mathrm{~nm})$ and pore volume $(1.12$ $\mathrm{cm}^{3} / \mathrm{g}$ ). The employment of nonpolar solvents (such as hexane) instead of water as the heating media plays a decisive role in the phase transformation. A post solvothermal procedure induced a phase transition from $2 \mathrm{D}$ hexagonal to $3 \mathrm{D}$ bicontinuous cubic FDU-5 mesostructure. The beginning materials are the mesostructured silica membranes prepared by the EISA method. The main reasons for the phase transition are the low polymerization degrees of inorganic silicate frameworks, the relatively high contents of organic templates, and the flexible nature of hybrid matrices.

\section{Synthesis of mesoporous alumina}

Based on the synthesis strategy, the synthesized mesoporous alumina may possess amorphous walls or crystalline walls. Alumina exists in different crystalline phases such as $\gamma, \eta, \delta$, and $\alpha . \gamma-\mathrm{Al}_{2} \mathrm{O}_{3}$ transforms to $\alpha-\mathrm{Al}_{2} \mathrm{O}_{3}$ when heated at higher temperature. However, collapse of mesoporous framework walls was observed at elevated temperatures. $\gamma-\mathrm{Al}_{2} \mathrm{O}_{3}$ can be obtained by heat treatment of $\mathrm{Al}(\mathrm{OH})_{3}$ or $\mathrm{AlOOH}$ at intermediate temperatures. Several researchers have reported different synthetic routes for preparation of mesoporous alumina. Some of the typical methodologies have been discussed below.

Baumann et al. ${ }^{(29)}$ and Hao et al. ${ }^{(30)}$ have used aluminum salts, propylene oxide and ethanol to prepare $\gamma-\mathrm{Al}_{2} \mathrm{O}_{3}$ through calcination at $800^{\circ} \mathrm{C}$. Vaudry et al.

${ }^{(31)}$ have reported the preparation of mesoporous aluminas using $\mathrm{Al}(\mathrm{sec}-\mathrm{BuO})_{3}$ as

Egypt. J. Chem. 59, No. 2 (2016) 
aluminum source, alcohol as solvent and different carboxylic acids as structure directing agents.

Liu et al. ${ }^{(32)}$ have described the synthesis of ordered crystalline molecular sieves via nanocasting route using mesoporous carbon (CMK-3) as template, aluminium nitrate as aluminum precursor and ethanol as solvent. Liu $e t$ al. have prepared $\gamma$-alumina single crystal nano leaves via hydrothermal route using $\mathrm{AlCl}_{3}, \mathrm{NaOH}, \mathrm{CTAB}$, ethanol (solvent) at $160^{\circ} \mathrm{C}{ }^{(32)}$. mesoporous alumina synthesis have been reported using $\mathrm{Al}(\mathrm{sec}-\mathrm{BuO})_{3}, 1$-propanol, lauric acid and autoclaving the reaction mixture at $110^{\circ} \mathrm{C}$ for $24 \mathrm{hr}$. The precursor was calcined at $600^{\circ} \mathrm{C}$ for $5 \mathrm{hr}$.

Kim et al. ${ }^{(33)}$ have employed post-hydrolysis method for synthesis of $\gamma$ alumina using $\mathrm{Al}(\mathrm{sec}-\mathrm{BuO})_{3}$, stearic acid and sec-butyl alcohol. Yada et al. ${ }^{(34}$ have reported the use of dodecyl sulfate as surface directing agent. Cabrera et al. ${ }^{(35)}$ have used triethanolamine with $\mathrm{CTAB}$ for synthesis of organized mesoporous aluminas (OMA). Cejka et al. ${ }^{(36)}$ have used $\mathrm{Al}(\mathrm{sec}-\mathrm{BuO})_{3}$, lauric acid or stearic acid and 1-propanol for preparation of OMA.

Alvine et al. ${ }^{(37)}$ have electrochemically prepared mesoporous membranes using a two step anodization technique. Mesoporous alumina molecular sieves (MSU-X) were synthesized by using polyethylene oxide and aluminium alkoxide. A neutral synthesis route for mesoporous alumina using triblock copolymer was described by Deng et al. ${ }^{(38)}$. A sol-gel synthesis route for mesoporous alumina has been reported using aluminium tri-tert butoxide, Pluronic P123, ethyl alcohol (solvent). Naik et al. ${ }^{(39)}$ have developed an aqueous solution based method for synthesis of mesoporous alumina by using was controlled by varying the chain length of carboxylic acid.

\section{Mechanisms of formation of mesostructured silicates}

In pursue to explain the mechanistic route of mesostructure formation several models have been proposed. At basic level, all these models suggest that, surfactant supramolecular assemblies provides template structure and this finally leads to the formation of pore structure in the inorganic matrix. Considering different mode of interaction of inorganic precursors with surfactants, several models have been proposed. Surfactant molecules are made-up of two distinct entities within the same molecule: hydrophilic head group and hydrophobic tail group. In aqueous medium, these molecules act as surface active agents and change the structural orientation depending on their concentration.

At low concentration, surfactant molecules exist as monomolecules. When the concentration of surfactant increases, they aggregate together to form micelles. The micelle concentration threshold, at which surfactant molecules aggregate to form isotropic micelles is called "critical micellization concentration" (CMC). Further increase in concentration results in formation of hexagonal closed packed arrays. Next step in this process is the coalescence of adjacent, mutually parallel cylinders to form lamellar phase. The cubic phase generally consists of complex, interwoven 
networks of rod shaped aggregates. Kresge et al. and Beck et al. ${ }^{(40,41)}$ have proposed liquid crystal templating mechanism for synthesis of M41S materials based on resemblance between lyotropic phases and M41S. The factors which influence the mesostructures are hydrocarbon chain length of surfactant molecules, concentration of surfactant and presence of organic swelling agents. For MCM-41 two mechanistic pathways have been proposed (I) liquid crystal templating mechanism and (II) charge density matching mechanism (Fig. 4).

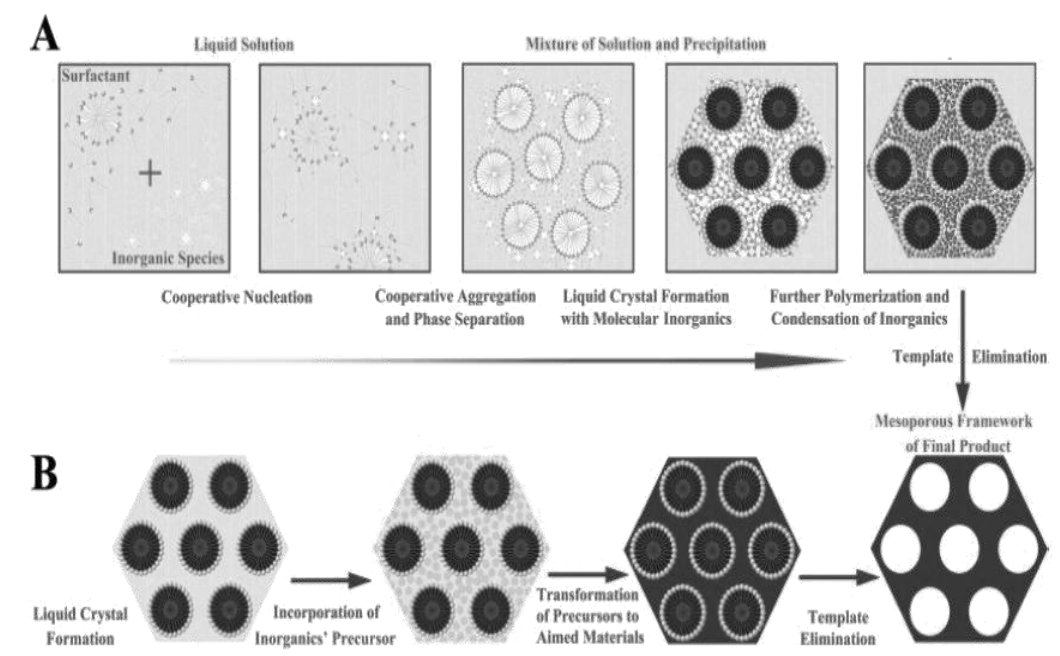

Fig. 4. Two mechanistic pathways of mesoporous materials: (A)Cooperative selfassembly; (B) "true" liquid-crystal templating process.

Pathway 1: Liquid crystal templating mechanism

This mechanism was proposed based on the similarity between liquid crystalline surfactant assemblies (i.e., lyotropic phases) and M41S. Two mechanistic pathways were postulated by the Mobil researchers:

1) Surfactant in solution forms lyotropic liquid crystal phase (LC). Deposition of inorganic precursors occurs on the micelle rods of preformed liquid crystalline phase. Subsequent polymerization of inorganic precursors leads to the formation of organic-inorganic mesostructure. This can be viewed as hexagonal array of surfactant micellar rods embedded in silica matrix. Removal of surfactants produces open, mesoporous framework.

2) Ordering of surfactant molecule into hexagonal liquid crystal phase is influenced by inorganic precursors. This was postulated as a cooperative self assembly of ammonium surfactants and silicate precursors below CMC.

Egypt. J. Chem. 59, No. 2 (2016) 
Chen et al. ${ }^{(42)}$ investigated in situ ${ }^{14} \mathrm{~N}$ NMR spectroscopy study of synthesis of MCM-41 under the same synthetic conditions reported by Mobil scientists and the results confirmed that LC did not form during MCM-41 synthesis. They proposed that, silicate deposition occurred on the surfactant micelle rods which were randomly arranged, followed by ordering of silicate deposited rods into hexagonal mesostructures. Aging of these mesostuctures at a favorable temperature helped in condensation of silicates into the MCM-41. However, according to Steel et al. ${ }^{(43)}$ silicate precursors directed the surfactant molecules into hexagonal LC phase. This result was supported by ${ }^{14} \mathrm{~N}$ NMR spectroscopy study. It was postulated that, the silicate precursors were present as organized layers in between the rows of the cylindrical miceller rods. Puckering of layers around the rods resulted in formation of hexagonal mesoporous structures.

Pathway 2: Charge density matching mechanism

Stucky et al. ${ }^{(4)}$ have introduced a new concept, Charge Density Matching Mechanism, to explain the mechanism of mesostructure formation.

From X-Ray diffraction study, it was confirmed that during synthesis the initial phase formed was layered structured. This was due to the electrostatic force of attraction between oppositely charged cationic surfactant head groups and anionic precursor species. Subsequent condensation step caused reduction in charge density of silicate species, which forced them to balance the charge density by introducing curvature into layers. As a result, lamellar structure transformed to hexagonal mesophase.

Apart from these two models, another model was proposed by Firouzi et al. (45), known as "silica tropic liquid crystals". They investigated the effect of electrolyte on miceller phase transitions through ${ }^{1} \mathrm{H},{ }^{29} \mathrm{Si}$ NMR spectroscopy and neutron scattering study of $\mathrm{CTAB}$ phase transition. $\mathrm{CTAB}$ transformed to hexagonal phase in presence of silicate ions. It was also proved that, in addition to charge balance requirement, the preferential bonding of ammonium group to the $\mathrm{D}_{4} \mathrm{R}$ silicate anions occurred under high $\mathrm{pH}$ condition. Silicate precursors, which were negatively charged ions, ion-exchanged with surfactant halide counter ions and produced "silica tropic liquid crystals", phase (SLC). Heat treatment of SLC resulted in irreversible condensation of silicates and formation of MCM-41. SLC phase displayed same characteristic features of lyotropic systems at low surfactant concentration. Huo et al. ${ }^{(46)}$ presented a generalized model to explain the interaction of surfactant head group (S) and inorganic precursor (I) as shown in Figure 5. This liquid crystal mechanism could be classified as $\mathrm{S}^{+} \mathrm{I}^{-}$pathway, where charge density matching occurred between oppositely charged surfactant and inorganic silicate ions through $\mathrm{SI}^{+}$pathway (where surfactant was anionic and inorganic precursors were positively charged). Charge interaction pathways could also proceed through same charge surfaces by $\mathrm{S}^{+} \mathrm{XI}^{+}$and $\mathrm{S}^{-} \mathrm{M}^{+} \mathrm{I}^{-}\left(\mathrm{M}^{+}\right.$is metal cation and $\mathrm{X}^{-}$is counter anion) pathways. Oppositely charged counter ions helped to neutralize the repulsion between same charged surfaces by serving as a charge balancing intermediates though hydrogen bonding. 
a)

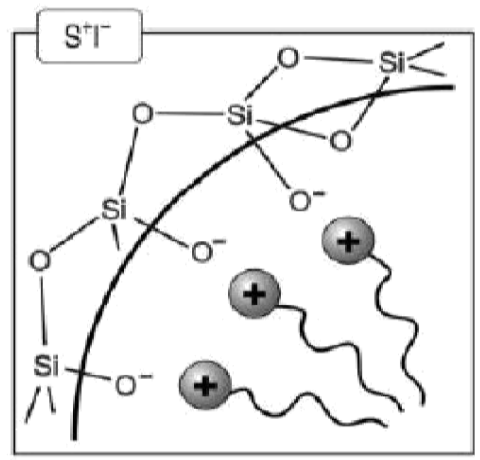

c)

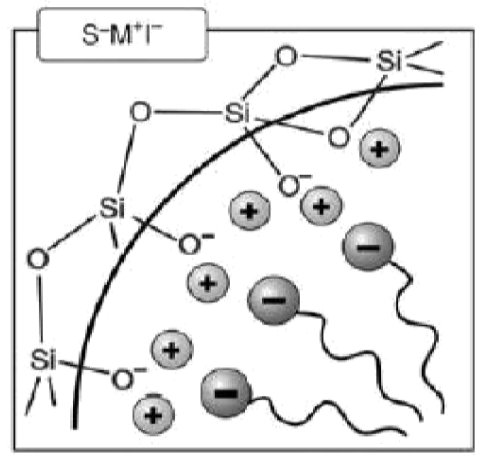

b)

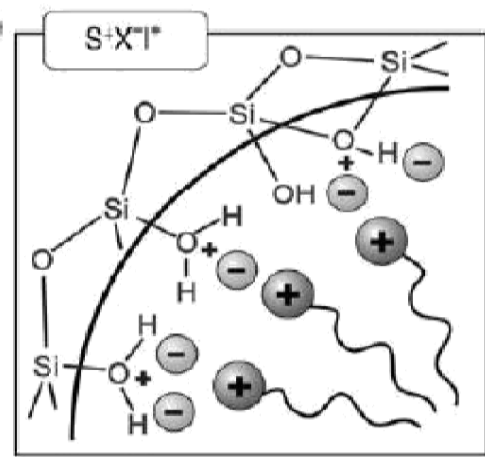

d)

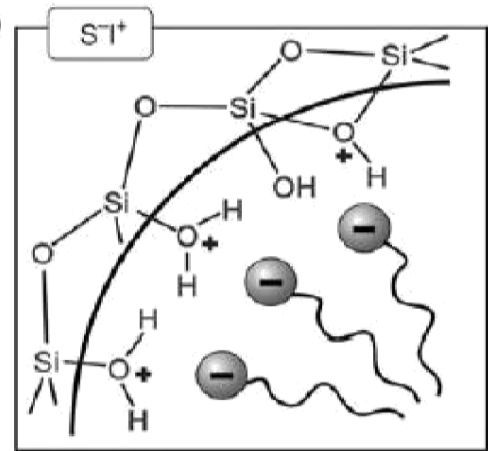

e)

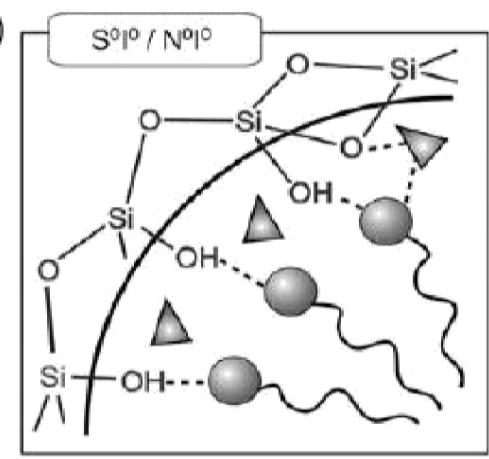

f)

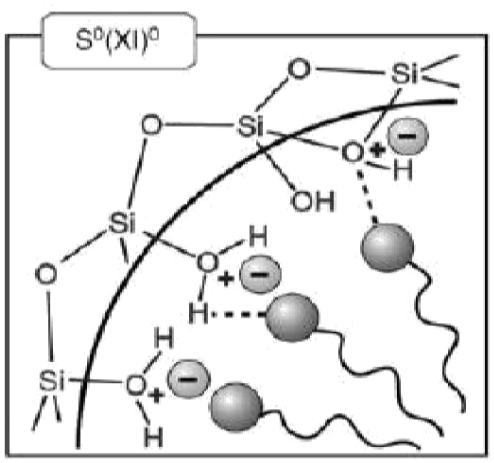

Fig. 5. Interactions between the inorganic species and the head group of the surfactant with consideration of the possible synthetic pathway in acidic, basic, or neutral media. Electrostatic: $\mathrm{S}^{+} \mathrm{I}^{-}, \mathrm{S}^{+} \mathrm{X}^{-} \mathrm{I}^{+}, \mathrm{S}^{-} \mathrm{M}^{+} \mathbf{I}^{-}, \mathrm{S}^{-} \mathrm{I}^{+}$; through hydrogen bonds: $\mathrm{S}^{0} \mathrm{I}^{0} / \mathrm{N}^{0} \mathrm{I}^{0}, \mathrm{~S}^{0}(\mathrm{XI})^{0}$.

\section{Formation of M41S family}

Silicate mesophases of M41S family of molecular sieves, mainly MCM-41, MCM-48 and MCM-50, derived from basic medium via $\mathrm{S}^{+} \mathrm{I}^{-}$route and by variation of surfactant to silicon molar ratio. MCM-41 possesses hexagonal Egypt. J. Chem. 59, No. 2 (2016) 
mesosphere belonging to $\mathrm{p} 6 \mathrm{~m}$ space group, MCM-48 structure shows cubic mesophase, which can be visualized as a two interlinked networks of spherical cages separated by continuous silicate frameworks. MCM-50, in uncalcined form, shows lamellar structure (p2), but after surfactant removal and post treatment, results into pillared layer material. Formation of phases of M41S is dependant on surfactant to silicon molar ratio. MCM-41, MCM-48 and MCM-50 forms when this ratio is $<1,1-1.5$ and $1.2-2$, respectively.

\section{Formation of SBA- $n$ materials}

SBA-1 and SBA-3 are generally synthesized by using cationic surfactants in acidic medium, which is below the isoelectric point of silica, through $\mathrm{S}^{+} \mathrm{XI}^{-}$pathway. These materials are termed as acid prepared materials (APM). SBA-1 is cubic structure but not bicontinuous like MCM-48, whereas, SBA-3 is hexagonal structure (p6m) similar to that of MCM-41. These materials are having thicker pore walls and the framework charge is different from that of base derived materials. This is due to the different precipitation conditions and charge balance system.

One of the important SBA group materials is SBA-15, which is different from other members of this family. It can be synthesized in acidic medium with non ionic pluronic surfactant ${ }^{(2)}$. SBA-15 has hexagonal structure similar to MCM41. SBA-16 is another material, synthesized with pluronic surfactant F127. These materials are synthesized in the highly acidic mediums with cationic silica precursor. At low $\mathrm{pH}$, protonated PEO chains of surfactants associate with cationic silica species though weak electrostatic interactions and pathway proceeds through $\mathrm{S}_{0} \mathrm{H}^{+} \mathrm{XI}^{+}$route. Silica, prepared with non ionic surfactants, posses thicker pore walls, due to slower self-assembly of silica-surfactant mesophase in gel compared to ionic surfactants.

Kim and Stucky ${ }^{(47)}$ reported the synthesis of highly ordered two dimentional hexagonal SBA-15, three dimentional hexagonal SBA-12 and cubic SBA-16 and SBA-11 using sodium matasilicate as silica source and nonionic amphiphilic block copolymers as structure directing agents.

\section{Mechanism of formation of mesoporous alumina}

The synthesis route employed by Davis et al. ${ }^{(30)}$ was hydrolysis of aluminium alkoxides in presence of carboxylate surfactant. In this method S-I complexation reaction between surfactant (S) and inorganic precursor (I) was involved. Hydrolysis of aluminium alkoxides took place by one of the two following reactions given below:

$$
\begin{array}{lll}
\mathrm{Al}(\mathrm{OR})_{3}+3 \mathrm{H} 2 \mathrm{O} & \longrightarrow & \mathrm{Al}(\mathrm{OH})_{3}+3 \mathrm{ROH}(1) \\
\mathrm{Al}(\mathrm{OR})_{3}+2 \mathrm{H} 2 \mathrm{O} & \longrightarrow & \mathrm{AlO}(\mathrm{OH})+3 \mathrm{ROH}(2)
\end{array}
$$

Bayerite $\left(\mathrm{Al}(\mathrm{OH})_{3}\right)$ was the final phase formed when hydrolysis was 
conducted in water $\left(\mathrm{Al}_{3}^{+}: \mathrm{H}_{2} \mathrm{O}>20\right)$ at a temperature $<350^{\circ} \mathrm{C}$. It was observed that, bayerite phase did not form when hydrolysis was performed in presence of carboxylic acid and large excess of water at $25^{\circ} \mathrm{C}$ for $24 \mathrm{hr}$. So presence of carboxylic acid inhibited the formation of bayerite phase. Pseudoboehmite was the major species formed in Vaudry-Davis method ${ }^{(30)}$. Here, assembly of carboxylic acidic coordinated with small $\mathrm{Al}(\mathrm{O}(\mathrm{OH})$ clusters formed and resulted in formation of mesophase consisting of organic core and inorganic shell.

Yada et al. ${ }^{(48)}$ proposed $\mathrm{SI}^{+}$assembly pathway when starting materials were dodecyl sulfate and aluminium nitrate. Zhang and Pinnavaia synthesized mesoporoue alumina (MSU-X) by $\mathrm{N}_{0} \mathrm{I}_{0}$ assembly, using electrically neutral polyethylene oxide surfactants $\left(\mathrm{N}_{0}\right)$ and aluminium alkoxides $\left(\mathrm{I}_{0}\right)$. In this method pore structure was formed via supramolecular assembly process. Liu et al. ${ }^{(49)}$ synthesized mesoporous crystalline alumina by using boehmite sols in presence of non-ionic surfactant (P123). They have proposed the possibility of a different mechanistic pathway other than widely accepted supramolecular self-assembly process. Unusual results of large surface area accompanied by high pore volume and large pore size in boehmite-P123 system were not observed before for mesoporous aluminas. Characterization results revealed that, weak interaction between precursor and P123 molecules had negligible influence on morphology of crystalline structure of boehmite. But, this interaction had capability to induce change in the stacking mode of boehmite layers as building blocks. This mechanism was more closely analogous to boehmite-surfactant sandwich structure proposed by González-Peña et al. ${ }^{(50)}$. Cejka et al. ${ }^{\left({ }^{(3)}\right)}$ reported the synthesis of organized mesoporous aluminas (OMA) by using carboxylic acids surfactants (lauric acid and stearic acids). Length of the hydrophobic hydrocarbon chain of carboxylic acids played the similar role like alkyl trimethylammonium surfactants in the synthesis of MCM- 41, i.e. increase of pore size with increasing chain length.

\section{Factors influence the structure of mesoporous materials \\ Effect of surfactant}

Surfactant chemistry is the fundamental subject matter in the study for mesoporous material synthesis and also in understanding the detailed mechanism of formation of mesoporous structures. The nature of surfactant governs the pore size, structure of pores, thickness of walls, phase and symmetry of materials. Surfactant molecules consist of hydrophilic polar head group and hydrophobic nonpolar hydrocarbon tail. Due to this amphiphilic nature, the surfactant molecules behave as active agents in aqueous solution showing high affinity to the surfaces and interfaces.

Depending on the nature of surfactant polar head group, surfactants are classified as anionic, cationic, zwitterionic and non ionic surfactants. At CMC, surfactant molecules self organize into micelles aggregates. Further increase in surfactant concentration or addition of some polar compounds results in their transformation to liquid crystalline phases. Structures of mesoporous materials

Egypt. J. Chem. 59, No. 2 (2016) 
are related to the shape and concentration of surfactant and can be expressed in terms of simple expression of surfactant packing parameter , $\mathrm{g}^{\mathrm{ee}}$, for lyotropic LC phase. $\mathrm{g}=\mathrm{V} / \mathrm{al}$, where $\mathrm{V}$ : is volume of surfactant tail, and a: is effective head group area and l: is length of the extended surfactant tail. Value of $g$ differs for different surfactants. This is due to the variation of different types of surfactant tails and heads groups. Hence, relative sizes of surfactant tail and head group are governing factors for optimum packing of the surfactants into different geometrical structures. $\mathrm{g}$ less than $1 / 3$, produces spherical micelles, further increase in concentration produces cubic phase, when $g=1 / 3$. When the value of $\mathrm{g}$ is $1 / 2$ hexagonal arrays form. When $\mathrm{g}$ value lies in between $1 / 3$ and $1 / 2$ formation of cubic phase occurs. Lamellar structure forms when $\mathrm{g}$ is equal to 1 . The effect $g$ value was observed in synthesis SBA-1 and SBA-3. When cationic surfactants are used in acidic medium, at $\mathrm{g}=1 / 3 \mathrm{SBA}-1$, having cubic phase, forms. But hexagonal phase forms when $\mathrm{g}=1 / 2$. Surfactants with larger head group (such as $\mathrm{C}_{\mathrm{n}} \mathrm{H}_{2 \mathrm{n}+1}\left(\mathrm{C}_{2} \mathrm{H}_{5}\right)_{3} \mathrm{~N}^{+}, \mathrm{n}=12,14,16,18$ ) favor the synthesis of SBA-1 in the acidic medium. Larger head groups decrease the value of surface packing parameter in order to generate maximum surface curvature. SBA-3 forms when head group of surfactant changes from $\mathrm{C}_{2} \mathrm{H}_{5}$ to $\mathrm{CH}_{3}$.

Mobil scientists produced three types of mesoporous silica (MCM-41, MCM48 and MCM-50) by varying the concentration of CTAB surfactant in basic $\mathrm{pH}$. $\mathrm{C}_{\mathrm{n}} \mathrm{H}_{2 \mathrm{n}+1}\left(\mathrm{CH}_{3}\right)_{3} \mathrm{~N}^{+}$with $\mathrm{n}=12,13,14,15,16,18$ can also be used for synthesis of MCM-41. Chiral surfactants $\left(\mathrm{C}_{16} \mathrm{H}_{33} \mathrm{~N}^{+}\left(\mathrm{CH}_{3}\right)_{2} \mathrm{CH}\left(\mathrm{CH}_{3}\right) \mathrm{C}_{6} \mathrm{H}_{5}\right)$ was also applied for production of MCM-41 and MCM-50. Zhao et al ${ }^{(10)}$. synthesized hexagonal SBA-15 with nonionic P123 ((EO) $\left.{ }_{20}(\mathrm{PO})_{70}(\mathrm{EO})_{20}\right)$ block copolymer in acidic medium. Use of Gemini surfactant was reported for synthesis of high quality mesoporous silica with cubic geometry. Gemini surfactant is known for self assembling at lower concentration compared to monomeric surfactants. Nonionic surfactants and partially ionized silica were used in neutral $\mathrm{pH}$ to obtain worm like mesostructures with mono dispersed pore size.

\section{Effect of $p H$}

$\mathrm{pH}$ is the important parameter which influences the synthetic chemistry of mesoporous materials. This is because of $\mathrm{pH}$ of medium, which influences charge of inorganic precursor species and surfactant head groups and consequently their mutual interaction. M41S family of molecular sieves was synthesized in basic $\mathrm{pH}$ by using CTAB, whereas Zhao et al. ${ }^{(10)}$ synthesized SBA materials in acid medium.

Due to high $\mathrm{pH}$ value, negatively charged inorganic precursors preferentially interacted with positively charged ammonium head groups of the surfactants and resulting in condensation of inorganic silicate ions into solid organicinorganic mesoporous structure. In case of SBA-15, at low $\mathrm{pH}$ polyethylene oxide chains get protonated and interact with positively charged silica species mediated by negatively charged halide ions to form $\mathrm{S}_{0} \mathrm{H}^{+} \mathrm{XI}^{+}$intermediate ${ }^{(2)}$.

Using the same surfactant different mesophases can also be obtained by Egypt. J. Chem. 59, No.2 (2016) 
changing the $\mathrm{pH}$ of reaction medium from acidic to basic. Change of $\mathrm{pH}$ values leads to phase transformation of silica from lamellar to hexagonal phase. Mechanism of formation of mesophases at acidic and basic extremes is completely different. This is because negatively charged silicate ions act as counter ions above isoelectric point $(\mathrm{pH} 2.0)$. Whereas below isoelectric point, positively charged silicate ions act as co-counter ions in acidic medium. Generally, in strong acidic medium rate of hydrolysis of TEOS is faster than condensation. Therefore, well ordered hexagonal arrangement of mesopores is obtained at acidic $\mathrm{pH}$, whereas the rate of condensation is faster than hydrolysis in neutral or basic conditions. Thus, $\mathrm{pH}$ plays a critical role in hydrolysis and condensation of inorganic silica species. Hydrolysis and condensation of silicon alkoxides can be expressed by the following three equations.

Hydrolysis: $\equiv \mathrm{Si}-\mathrm{OR}+\mathrm{H}_{2} \mathrm{O} \longrightarrow \quad \longrightarrow \mathrm{Si}-\mathrm{OH}+\mathrm{ROH}$
Alcohol condensation: $\equiv \mathrm{Si}-\mathrm{OR}+\mathrm{SI} \equiv \longrightarrow \mathrm{Si}-\mathrm{O}-\mathrm{Si} \equiv+\mathrm{ROH}$
Water condensation: $\equiv \mathrm{Si}-\mathrm{OH}+\mathrm{HO}-\mathrm{Si} \equiv \longrightarrow \mathrm{Si}-\mathrm{O}-\mathrm{Si} \equiv+\mathrm{H}_{2} \mathrm{O}$

When $\mathrm{pH}$ is 2 , charge on silica becomes zero and $\mathrm{pH}$ above 2 , the silica species $[\mathrm{Si}(\mathrm{OH}) 4]$ condense into small silica aggregates, as shown in equation $\mathrm{c}$ and have negative charge on the surface due to silanol groups. It has been observed that, slow growth rate is the fundamental requirement for formation of crystals with well shape-morphology. Silica condensation is slowest and surface charge is electronically neutral around isoelectric point at $\mathrm{pH}=2 . \mathrm{pH}$ dependency of condensation rate was exhibited by SBA-1 when its crystal shape showed transitions from sphere $\longrightarrow$ decaoctahedron $\longrightarrow$ truncated cube $\longrightarrow$ cube with change in $\mathrm{pH}$ from 1.0 to 2 . The electrolyte concentration is also a factor which effects mesophase formation.

The effect of amines on the distribution of silica and aluminosilicate species was observed in the synthesis of mesoporous silica. Solubility and distribution of silicate structures can be altered by addition of organic alkyl ammoniums to the silica source. Commonly tetramethylammonium hydroxide (TMOH) is used to dissolve silica. Major forms of silica structures produce are D3R(Q36), D4R(Q38) and D5R(Q310).

Firouzi et al. ${ }^{(45)}$ showed that there is a preferential bonding of ammonium head group of alkyl trimethylammonim surfactant to D4R silicate anions at high $\mathrm{pH}$. This was proved by the formation of $\mathrm{D} 4 \mathrm{R}$ in solution of silicate anions which did not contain D4R species. Alkyl trimethylammonim surfactant forced non D4R species to re-equilibrate and formed D4R. One of the suggestions for this observation was that the projected areas of D4R anion and ammonium head group were matching.

\section{Effect of silica source}

Mesoporous silica synthesis is vibrant with respect to source of silica used. Mesoporous structures have been synthesized by making use of several sources of silica, such as silicon alkoxides (e.g., TEOS, TMOS), sodium silicate, Ludox,

Egypt. J. Chem. 59, No. 2 (2016) 
fumed silica, water glass etc. Silicon alkoxides are not soluble in water but can be dissolved in a mixture of water and water miscible organic solvent (e.g., ethanol). They can be hydrolyzed by acid catalyzed or base catalyzed reaction resulting in cleavage of ester bond. They condense into aggregates containing $\mathrm{Si}-\mathrm{O}-\mathrm{Si}$ species and have negative charge due to silanol groups. In TEOS and other simple alkoxides, number of siloxane bonds is zero. Thus, TEOS shows 29Si-NMR $\mathrm{Si}(\mathrm{Qn})$ signal to be $\mathrm{Si}(\mathrm{Q} 0)$, where value of $\mathrm{n}$ represents coordination number of silicon with siloxane bonds. Colloidal silica shows $\mathrm{Si}(\mathrm{Q} 4)$ whereas water glass can exhibit maximum of five signals.

It is found that, silica sources displaying $\mathrm{Si}(\mathrm{Q} 4)$ signals are unsuitable as starting material in synthesis of mesoporous silica. Klotz et al. ${ }^{(51)}$ showed that for the formation of ordered mesoporous materials require $\mathrm{Si}(\mathrm{Q} 1)$ species in the initial stage of synthesis. Presence of $\mathrm{Si}(\mathrm{Q} 3)$ species in initial stage of the synthesis resulted in disordered mesoporous silica. Setoguchi et al. ${ }^{(52)}$ studied the effect of various silica sources under strong acidic conditions $(\mathrm{pH}<1)$ with $\mathrm{C}_{6} \mathrm{PyCl}$ surfactant. They found that use of sodium silicate produced high quality mesoporous silica analogous to MCM-41, whereas use of as received colloidal silica resulted in formation of non-mesoporous silica ${ }^{(52)}$. Mesoporous silica produced from water glass also showed interesting results. Type I water glass produced mesoporous silica but type III did not. Setoguchi et al. ${ }^{(52)}$ have also synthesized mesoporous silica using Huo et al. ${ }^{(46)}$ method. TEOS produced mesoporous silica with high surface area and narrow pore size distribution. This can be compared with mesoporous silica, prepared by using sodium silicate. Kim et al. ${ }^{(33)}$ showed that in rapid hydrolysis condition TMOS acted as preferential precursor source than TEOS.

\section{Characterization of ordered mesoporous materials}

$X$-ray diffraction (XRD)

$\mathrm{X}$-ray diffraction is one of the most important and most powerful methods for the investigation of materials. This method is based on the scattering of X-rays by the electrons of atoms. The wavelengths of X-rays are similar to interatomic distances and so the X-rays scattered by different atoms will interfere destructively or constructively, in the latter case giving rise to diffracted beams. In the case of crystalline samples, sharp diffraction phenomena result. The geometry of the corresponding diffraction events can be described by Braggee $\mathrm{s}$ law, which combines a measure of the lattice of the crystal structure, namely the distance $\mathrm{d}$ between lattice planes, the wavelength $\lambda$ of the $\mathrm{X}$-ray radiation and the diffraction angle $\theta: \lambda=2 \mathrm{~d} \sin \theta$ : The Bragg's equation treats diffraction as the reflection of X-rays at the lattice planes; correspondingly, a diffraction event is usually called a reflection. Figure 6 shows XRD pattern for SBA-15 materials (p6mm space group) with indices of the diffraction planes. The corresponding $\mathrm{d}$ spacings: $(100)=9.80 \mathrm{~nm} ;(110)=5.45 \mathrm{~nm}$ and $(200)=4.74 \mathrm{~nm}$. The XRD patterns for SBA-15 and Fe-SBA-15 samples as shown in Fig. 7 (a, b) in lowangle region show three well-resolved peaks at (100), (110) and (200), which are characteristic of mesoporous material with 2D-hexagonal structure. They resemble that one of the parent mesoporous silica SBA-15, indicating that the 
materials prepared have highly ordered mesoporous structures ${ }^{(10)}$. Moreover, in the wide-angle region the wide line of amorphous silica was observed, no diffraction peaks corresponding to crystalline iron oxides could be observed (Fig. 7b). Possibly, iron species were introduced into the framework of ordered mesoporous silica or the particle size of iron oxides in the sample was too small to be detected by $\mathrm{x}$-ray diffraction. The absence of these prominent reflections in case of the nanostructured $\mathrm{Fe}_{2} \mathrm{O}_{3}$ indicated that no crystalline bulk materials have been formed outside the pore system. The result also showed that $\mathrm{Fe}_{2} \mathrm{O}_{3}$ nanoparticles were dispersed uniformly in the frame of SBA-15 ${ }^{(52)}$. On the Other hand, the intensity of the line at d100 decreases, upon loading with Ni (Fig. 8a) this may indicate the penetration of the nickel oxide inside the mesopore system of AlSBA-15 support ${ }^{(18)}$.

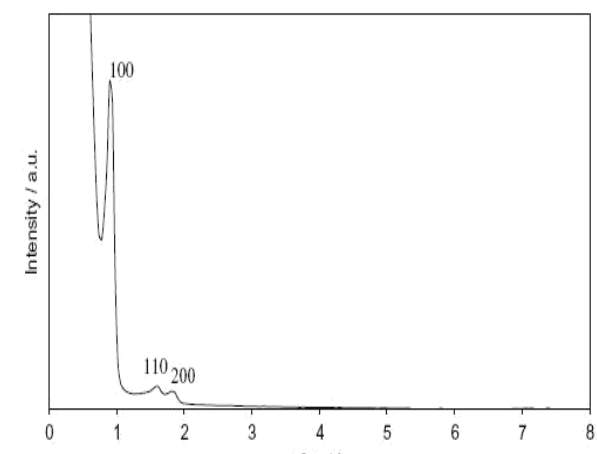

Fig. 6. Typical XRD pattern of SBA-15.

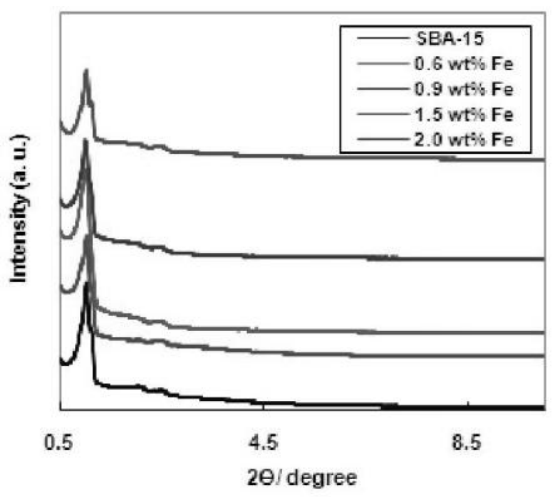

Fig. 7a. Low angle XRD of Fe-SBA-15 catalysts.

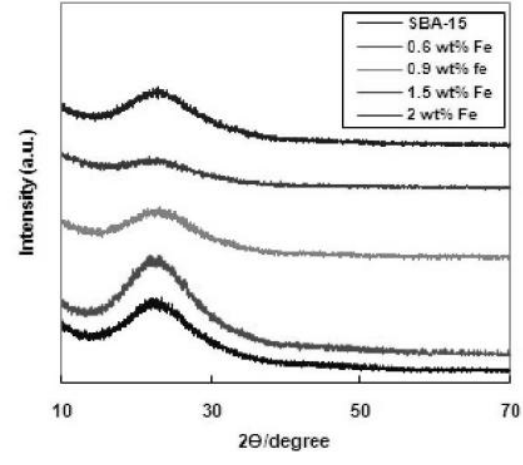

Fig.7b. High angle XRD of Fe-SBA-15 catalysts.

Egypt. J. Chem. 59, No. 2 (2016) 

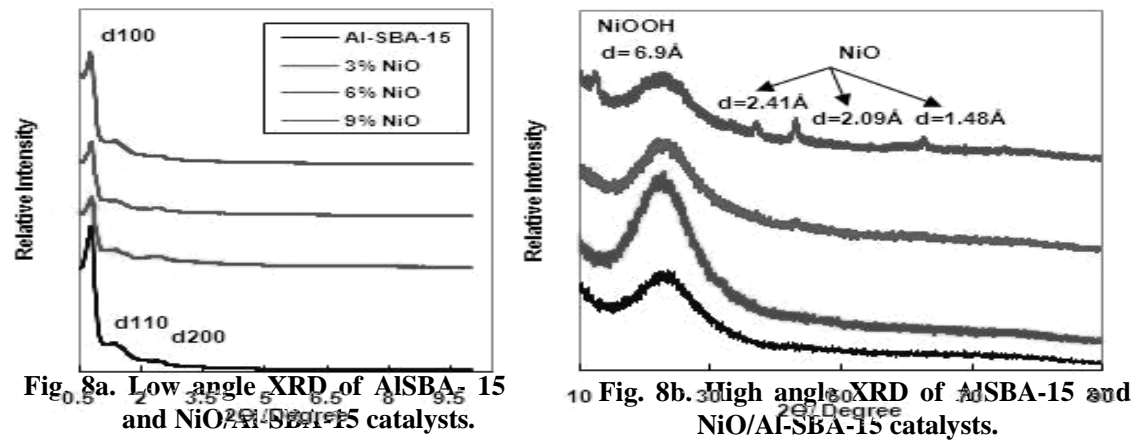

Moreover, in the wide-angle region, it is clear that; classification, with capillary condensation at medium relative pressure being indicative of mesoporosity. In some cases the desorption branch is different to the adsorption path, the isotherm then exhibiting a hysteresis loop. The shape of the hysteresis loop provides information about the shape and the size of the mesopores. $\mathrm{N}_{2}$ adsorption-desorption isotherms and BJH pore size distributions for SBA-15 and Fe-SBA-15 samples are illustrated in Fig. 9 ( $a$ and b). The deposition of Fe species on SBA-15 support did not modify greatly the isotherm shape of SBA-15 support $^{(53)}$.

\section{$\mathrm{N}_{2}$ adsorption- desorption}

The obtained isotherms for SBA-15 and Fe-SBA-15 samples are of type IV according to the IUPAC classification, being typical for mesoporous materials with 2D-hexagonal structure that have large pore sizes with narrowed pore size distributions ${ }^{(10)}$. The observed $\mathrm{H} 1$ type hysteresis loops are typical of mesoporous materials with uniform tubular pores. Such H1 type hysteresis loops indicate also the presence of open channels. The well-defined step, occurred at high relative pressures $(0.4-0.8)$, correspond to capillary condensation of $\mathrm{N} 2$, related to the uniformity of the pores.
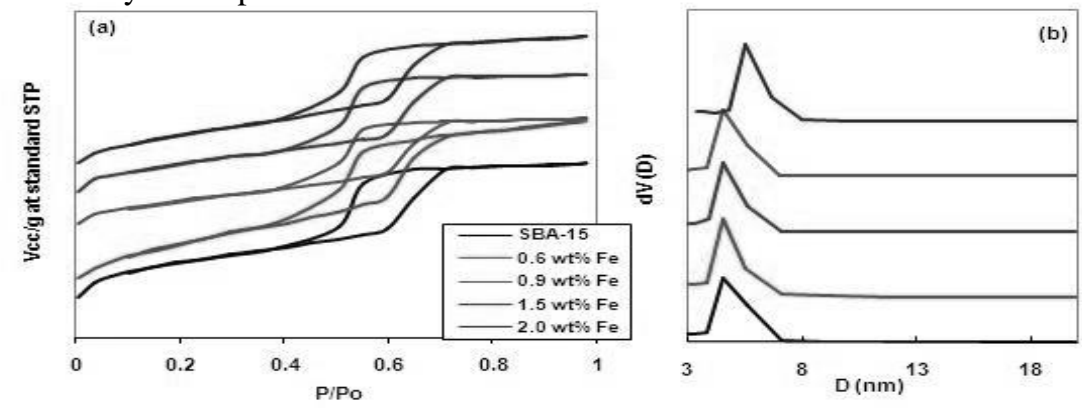

Fig.9. $\mathrm{N}_{2}$ adsorption-desorption isotherms (a) and pore size distribustion (b) of SBA-15 and Fe-SBA-15 samples.

Scanning electron microscopy (SEM) 
Scanning electron microscopy characterization is used primarily for the study of surface topography and morphology of solid materials on a scale down to about 10 $\mathrm{nm}$. Topographical features, void content, particle agglomeration as well as compositional and structural differences within the material can be revealed. The technique works on the principle that an electron beam is passing through an evacuated column and focused by electromagnetic lenses onto the material. The beam is scanned over the specimen surface in synchronism with the beam of a cathode ray tube (CRT) display screen. In elastically scattered secondary electrons are emitted from the sample surface and collected by a scintillator, the signal from which is used to modulate the brightness of the cathode ray tube. In this way the secondary electron emission from the sample is used to form an image on the CRT display screen. Differences in secondary emission result from changes in surface topography. If (elastically) backscattered electrons are collected to form the image, contrast results from compositional and structural differences and often diffraction patterns can be obtained for crystalline materials.

Materials can only be studied properly when they are electrically conducting, as the electrons otherwise give rise to charging phenomena resulting in blurred images. Non-conducting materials (amongst which silica-based materials) need to be sputtered with a thin layer of conducting material before being inserted into the SEM, and connected to a conducting sample holder. In general, $\mathrm{Au}$ or $\mathrm{C}$ is used for sputtering in a plasma sputter-coater.
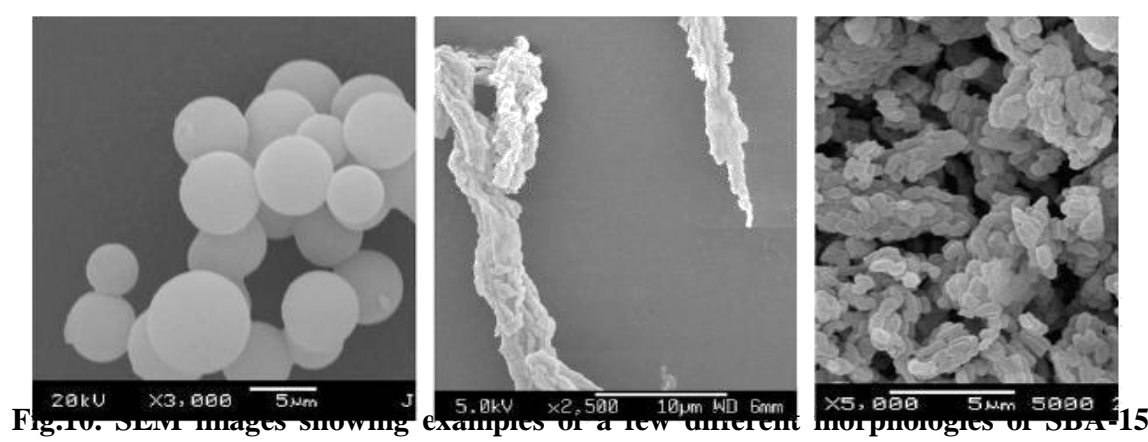

(spheres, fibers and rods).

\section{Transmission electron microscopy (TEM)}

The ultimate technique to obtain direct structural information at nanometer scale resolution for porous materials is transmission electron microscopy. Whereas in SEM the detectors are mounted on the same side of the sample as the impinging beam in order to detect the scattered secondary electrons, for TEM the detectors are mounted behind the sample to detect the electrons transmitted through a thin section (preferably less than $100 \mathrm{~nm}$ ) of the material. The image in TEM is the result of diffraction contrast.

The sample is oriented so that some of the beam is transmitted and some is

Egypt. J. Chem. 59, No. 2 (2016) 
diffracted out. Any local structural variation in the sample causes a different fraction of the incident beam intensity to be "diffracted out", leading to a variation in image darkness on a viewing screen at the base of the microscope. Magnification is achieved by using lenses underneath the sample to project the image formed by the diffracted electrons onto a recording device. The magnification is determined by the optical system and the resolution by the aberrations in the lens performance. Atomic resolution can be obtained for crystalline materials. Powders can be crushed lightly, to separate primary particles and supported on special, electrically conducting and partially electron transparent sample holders (TEM grids). Large particles of non-conducting material will show charging phenomena leading to blurred images.
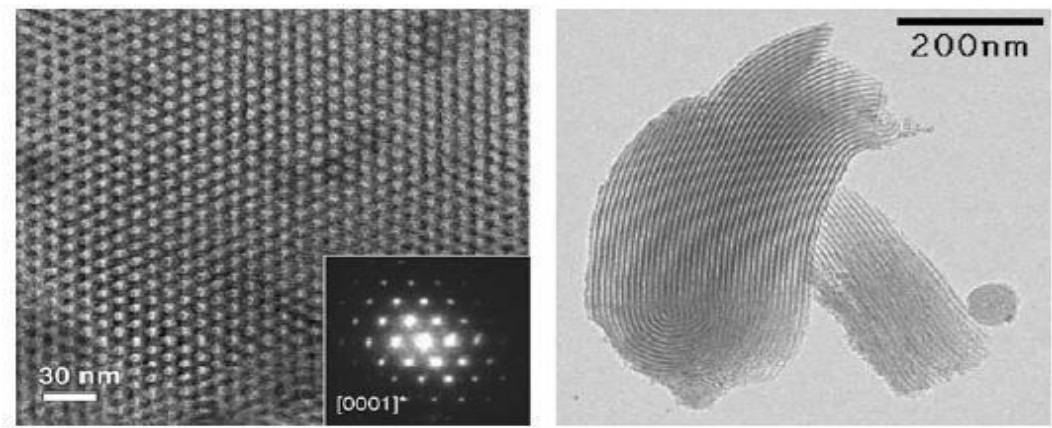

Fig.11. TEM images and diffraction pattern showing the hexagonal ordering of SBA15 and the curved pores.

Applications of the porous materials

Applications of porous silicates

Metal ion loaded M41S and SBA-15 based materials are having several applications such as, in heterogeneous catalyzed reactions (oxidation, acid catalyses, hydroxylation and polymerization), adsorbents, separation processes, host guest encapsulation, host of quantum structures etc. Some applications of various metal ion incorporates mesoporous silica are listed in Table 4.

\section{Applications of mesoporous aluminas}

High surface area aluminas with porous structure have gained importance due to their potential applications as catalysts and catalyst supports as well as adsorptive material in the separation processes. Favorable textural properties and intrinsic acid base properties have resulted in their extensive use as a catalyst supports. Transition alumina especially $\gamma$-alumina, has potential to be a major catalytic support in automotive, petroleum industries and in adsorption technology. The acidic function of the support accomplishes the isomerization and cyclization of normal paraffins, the supported metal convert low octane components to high-octane isoparaffins. In addition, micro and nanocrystalline aluminas are commonly used for $\mathrm{Al}_{2} \mathrm{O}_{3}$ - based ceramics. 
TABLE 3 . Applications of various mesoporous silicates.

\begin{tabular}{|c|c|c|}
\hline Materials & Application & Remarks \\
\hline NiMo-MCM-41 & $\begin{array}{c}\text { Catalysts for gas oil } \\
\text { hydrocracking. } \\
\text { NiMo-MCM-41 showed } \\
\text { higher } \\
\text { hydrodesulurization } \\
\text { and } \\
\text { hydrodenitrogenetaion } \\
\text { activity than NiMo loaded } \\
\text { on USY oramorphous } \\
\text { aluminosilicate. }\end{array}$ & $\begin{array}{l}\text { NiMo-MCM-41 showed higher } \\
\text { hydrodesulurization and } \\
\text { hydrodenitrogenetaion activity } \\
\text { than NiMo loaded on USY or } \\
\text { amorphous aluminosilicate. }\end{array}$ \\
\hline $\begin{array}{l}\text { Cr-MCM-41 and } \\
\text { Cr-MCM-48 }\end{array}$ & $\begin{array}{l}\text { oxidation of } \\
\text { ethylbenzene }\end{array}$ & $\begin{array}{l}\text { These catalysts showed quite } \\
\text { promising results for liquid } \\
\text { phase oxidation } \\
\text { ethylbenzene. }\end{array}$ \\
\hline Al-MCM-41 & $\begin{array}{l}\text { Diels-Alder reaction of } \\
\text { cyclopentadiene with } \\
\text { several unsaturated } \\
\text { aldehydes, }\end{array}$ & $\begin{array}{l}\text { These catalysts showed quite } \\
\text { promising results for liquid } \\
\text { phase oxidation } \\
\text { ethylbenzene. }\end{array}$ \\
\hline Al-MCM-41 & $\begin{array}{l}\text { Diels-Alder reaction of } \\
\text { cyclopentadiene with } \\
\text { several unsaturated } \\
\text { aldehydes, }\end{array}$ & $\begin{array}{l}\text { Al-MCM-41 catalyst showed } \\
\text { highest activity compared to } \\
\text { the HZSM-5 in all the reactions } \\
\text { tested. }\end{array}$ \\
\hline $\begin{array}{l}\text { Cobalt containing SBA- } \\
15\end{array}$ & Fischer-Tropsch reaction & $\begin{array}{l}\text { Highly dispersed Co in SBA- } \\
15 \text { exhibited high catalytic } \\
\text { activity which was attributed to } \\
\text { the ordered pore structure of } \\
\text { SBA- } 15 \text {. }\end{array}$ \\
\hline $\begin{array}{l}\text { Ni-SBA-15 and Ni- } \\
\text { SBA-16 }\end{array}$ & $\begin{array}{l}\text { Catalyst for selective } \\
\text { hydrodechlorination of } \\
\text { 1,1,2-trichloroethane }\end{array}$ & $\begin{array}{l}\text { Ni-SBA-16 sample showed } \\
\text { highest activity. This was due } \\
\text { to its three-dimensional pore } \\
\text { structure, which allowed rapid } \\
\text { diffusion of molecules through } \\
\text { the pore system. }\end{array}$ \\
\hline $\begin{array}{l}\text { Fe- and Pt- containing } \\
\text { MCM-41 }\end{array}$ & De-NOx reaction & $\begin{array}{l}\text { Promising results for de-NOx } \\
\text { reaction was observed. }\end{array}$ \\
\hline $\begin{array}{l}\text { Na-MCM-41 and Cs- } \\
\text { MCM-41 }\end{array}$ & Base catalysis & $\begin{array}{l}\text { In knoevenagel condensation of } \\
\text { benzaldehyde with ethyl } \\
\text { cyanoacetate, excellent } \\
\text { conversion of benzaldehyde } \\
\text { and selectivity was observed. }\end{array}$ \\
\hline Ti-MCM-41 & $\begin{array}{l}\text { Catalysts for } \\
\text { hydroxylation of benzene }\end{array}$ & $\begin{array}{l}\text { Excellent catalytic oxidation } \\
\text { performance in presence of } \\
\text { hydrogen peroxide. }\end{array}$ \\
\hline
\end{tabular}

Egypt. J. Chem. 59, No. 2 (2016) 
TABLE 3. Cont.

\begin{tabular}{|c|c|c|}
\hline Pt-MCM-48 & $\begin{array}{l}\text { Hydrogenation of benzene } \\
\text { and toluene }\end{array}$ & $\begin{array}{l}\text { This catalyst showed high } \\
\text { activity in the hydrogenation of } \\
\text { benzene and toluene. }\end{array}$ \\
\hline $\begin{array}{l}\text { Pt and Rh nanowires } \\
\text { and/or particles were } \\
\text { prepared in mesopores } \\
\text { of FSM-16 }\end{array}$ & $\begin{array}{l}\text { Hydrogenation and } \\
\text { isomerisation catalyst }\end{array}$ & $\begin{array}{l}\text { Under solvent-free and low } \\
\text { temperature condition showed } \\
\text { selective hydrogenation. } \\
\text { Superior performance by chiral } \\
\text { catalyst confined within } \\
\text { Mesoporous silica. }\end{array}$ \\
\hline $\begin{array}{l}\text { Bimetallic nanoparticles } \\
\text { were prepared from } \\
\text { Ru6Pd6 and Ru6CSn } \\
\text { carbonyl clusters were } \\
\text { anchored along the } \\
\text { interior walls of } \\
\text { mesoporous silica MCM- } \\
41\end{array}$ & $\begin{array}{c}\text { Hydrogenation and chiral } \\
\text { catalyst }\end{array}$ & $\begin{array}{l}\text { Under solvent-free and low } \\
\text { temperature condition showed } \\
\text { selective hydrogenation. } \\
\text { Superior performance by chiral } \\
\text { catalyst confined within } \\
\text { mesoporous silica. }\end{array}$ \\
\hline $\begin{array}{l}\text { Gold Nanoparticles in } \\
\text { SBA-15 }\end{array}$ & Catalyst for CO oxidation, & $\begin{array}{l}\text { SBA-15 was functionalized with } \\
\text { positively charged groups and } \\
\text { [AuCl4]- -species were } \\
\text { subsequently incorporated into } \\
\text { the channel system via ion } \\
\text { exchange. Upon reduction with } \\
\text { NaBH4, highly dispersed } \\
\text { nanoparticles of gold were } \\
\text { formed in the channels } \\
\text { of the mesoporous host. }\end{array}$ \\
\hline $\begin{array}{l}\text { Sulfide ion within } \\
\text { MCM-41 }\end{array}$ & $\begin{array}{l}\text { Catalytic reduction of } \\
\text { methyl viologen }\end{array}$ & $\begin{array}{l}\text { Methyl viologen in the presence } \\
\text { of mesoporous MCM- } 41 \text { and } \\
\text { sulfide ions is found to undergo a } \\
\text { spontaneous catalytic reduction } \\
\text { to methyl viologen radical } \\
\left(\mathrm{MV}^{+}\right) \text {. }\end{array}$ \\
\hline
\end{tabular}

\section{References}

1. Naik, B. and Ghosh, N.N., A review on chemical methodologies for preparation of mesoporous silica and alumina based materials. Recent Patents on Nanotechnology, $\mathbf{3}$, 213-224 (2009).

2. Wan, Y. and Zhao, D., On the controllable soft-templating approach to mesoporous silicates. Chem. Review 107(7), 2221- 2860 (2006).

3. Mu, J. He, Y. and Wang Y., Copper-incorporated SBA-15 with peroxidase-like activity and its application for colorimetric detection of glucose in human serum. Talanta, 148, 22-28 (2016).

4. He, S., Zhang, L., Li, X., Wang, J., He, D., Lua, J. and Luo Y., Hydrogen production by ethanol steam reforming over Ni/SBA-15mesoporous catalysts: Effect of Au addition.Catalysis Today, 258, 162-168 (2015).

Egypt. J. Chem. 59, No.2 (2016) 
5. Tomer, V.K. and Duhan, S., Sensors and Actuators, B: Chemical, Nano titania loaded mesoporous silica: Preparation and application as high performance humidity sensor, B 220,192-200 (2015).

6. Tomer, V.K., Vijay, K.T. and Duhan, S., Sensors and Actuators, A facile nanocasting synthesis of mesoporous Ag-doped $\mathrm{SnO}_{2}$ nanostructures with enhanced humidity sensing performance, B 223, 750-760 (2016).

7. Gaudina, P., Dorgeb, S., Noualia, H., Vierlingc, M., Fiani, E., Molièree, M., Brilhac, J.F. and Patarin J., CuO/SBA-15 materials synthesized by solid state grinding: Influenceof $\mathrm{CuO}$ dispersion and multicycle operation on DeSOX performances. Applied Catalysis B: Environmental, 181, 379-388 (2016).

8. Zhu, L., Qu, H., Zhang and Qiangfei, L., Direct synthesis, characterization and catalytic performance of Al-Fe-SBA-15 materials in selective catalytic reduction of $\mathrm{NO}$ with $\mathrm{NH}_{3}$. Catalysis Communications, 73, 118-122 (2016).

9. Ramos, J.M., Wang, J.A., Chen, L.F., Arellanoa, U., Ramírez, S.P., Sotelo, R. and Schachat, P., Synthesis and catalytic evaluation of CoMo/SBA-15 catalysts for oxidative removal of dibenzothiophene from a model diesel. Catalysis Communications, 72, 57-62 (2015).

10. Zhao, D.Y., Feng, J.L., Huo, Q.S., Melosh, N., Fredrickson, G.H., Chmelka, B.F. and Stucky, G.D., Triblock co-poymer syntheses of mesoporous silica with periodic 50 to 300 Angstrom pores. Science, 279, 548-552 (1998).

11. Cheng, Y.R., Lin, H.P. and Mou, C.Y., Control of mesostructure and morphology of surfactant-templated silica in a mixed surfactant system. Phys. Chem. Chem. Phys. 1, 5051-5058 (1999).

12. Ryan, K.M., Coleman, N. R.B., Lyons, D.M., Hanrahan, J.P., Spalding, T.R., Morris, M.A., Steytler, D.C., Heenan, R.K. and Holmes, J.D., Control of pore morphology in mesoporous silicas synthesized from triblock copolymer templates, Langmuir, 18, 4996-5001(2002).

13. Lee, J., Yoon, S., Hyeon, T., Oh, S.M. and Kim, K.B., Synthesis of a new mesoporous carbon and its application to electrochemical doublelayer capacitors. Chemical Communications. 21, 2177-2178 (1999).

14. Jin, Z., Wang, $\mathbf{X}$. and Cui, X., A two-step route to synthesis of small-pored and thick-walled SBA-16-type mesoporous silica under mildly acidic conditions. $J$. Colloid Interf. Sci. 307, 158-165 (2007).

15. Voegtlin, A.C., Ruch, F., Guth, J.L., Patarin, J. and Huve, L., Synthesis of mesoporous silica with ionic- and non-ionic surfactants a new templating pathway. Micropor. Mater. 9, 95-105 (1997).

16. Schmidt-Winkel, P., Yang, P., Margolese, D.I., Chmelka, B.F. and Stucky, G.D., Fluoride-induced hierarchical ordering of mesoporous silica in aqueous acidSyntheses. Adv. Mater. 11, 303- 307 (1999).

Egypt. J. Chem. 59, No. 2 (2016) 
17. Gobara H.M., Elsalamony R. A. and Hassan, S. A., Sonophotocatalytic Degradation of Eriochrome black-T Dye in Water using Ti Grafted SBA-15, $J$. Porous Mater. 23 (5), 1311-1318 (2016).

18. Gobara H.M., Characterization and catalytic activity of $\mathrm{NiO} / \mathrm{mesoporous}$ aluminosilicate AISBA-15 in conversion of some hydrocarbons. Egyptian Journal of Petroleum, 21(1), 1-10 (2012).

19. Yue Y., Gédéon A., Bonardet J.-L., Melosh N., Espinose J.B. D. and Fraissard J., Direct incorporation of A1 in SBA mesoporous materials: characterization, stability and catalytic activity, Stud. Surf. Sci. Catal. 129 , 209-218. (2000) .

20. Janicke, M., Kumar, D., Stucky, G.D. and Chemlka, B.F., Aluminium incorporation in mesoporous molecular sieves. Stud. Surf. Sci. Catal. 84,243-250 (1994).

21. Reddy, K.M. and Song, C., Synthsis of mesoporous molecular sieves: the influence of Aluminium source in $\mathrm{Al}$ incorporation in MCM-41. Catal. Lett. 36, 103 109 (1996).

22. Hartmann M. and Bischof C., Mesoporous Molecular Sieves, Stud. Surf. Sci. Catal. 117, 249- 257 (1998)

23. El Naggar, A.M.A., Gobara, H.M., EI Sayed, H.A. and Soliman, F.S., New advances in hydrogen frame-worked MoO3. Energy Conversion and Management, 106, 615-624 (2015)

24. Kresge, C.T., Leoniwicz, M.E., Roth, W.J., Vartuli, J.C. and Beck, J.S., Ordered mesoporous molecular sieves synthesized by a liquid-crystal template mechanism. Nature, 359,710-712(1992).

25. Huo, Q.S., Margolese, D.I., Ciesla, U., Feng, P.Y., Gier, T.E., Sieger, P., Leon, R., Petroff, P.M., Schuth, F. and Stucky G.D., Generalized synthesis of periodic surfactant inorganic composite-materials. Nature, 368. 317-321(1994).

26. Kleitz, F., Liu, D.N., Anilkumar, G.M., Park, I.S., Solovyov, L.A., Shmakov, A.N. and Ryoo R.J., Large cage face-centered-cubic $\mathrm{Fm}_{3} \mathrm{~m}$ mesoporous silica: synthesis and structure. Phys. Chem. B: 107, 14296-14300(2003).

27. Lu, Y.F., Ganguli, R., Drewien, C.A., Anderson, M.T., Brinker, C.J., Gong, W.L., Guo, Y.X., Soyez, H., Dunn, B., Huang, M.H. and Zink, J.I., Continuous formation of supported cubic and hexagonal mesoporous films by sol gel dip-coating. Nature, 389, 364-368 (1997).

28. Xia, Y.D. and aya, R.J., A study of the behaviour of mesoporous silicas in $\mathrm{OH} / \mathrm{CTABr} / \mathrm{H}_{2} \mathrm{O}$ systems: phase ependent stabilisation, dissolution or semipseudomorphic transformation. J. Mater. Chem. 13, 3112- 3121(2003).

29. Baumann, T.F., Gash, A.E. and Chinn, S.C., et al., Synthesis of high surfacearea alumina aerogel without use of alkoxide precursor. Chem Mater. 17, 395-401 (2005). 
30. Hao, Z.X., Liu, H.,Guo, B., Li, H., Zhang, J.W., Gan, L.H., Xu, Z.J. and Chen L.W., Sol- gel synthesis of alumina using inorganic salt precursor. Acta PhysChim. Sin. 23, 289-294 (2007).

31. Vaudry, F., Khodabandeh, S. and Davis, M., Tailoring the electronic structure of mesoporous spinel $\gamma-\mathrm{Al}_{2} \mathrm{O}_{3}$ at Atomic Level: $\mathrm{Cu}$-Doped Case. Chem Mater. 8, 1451-1464 (1996).

32. Liu, Y., Ma, D. and Han, X., Hydrothermal synthesis of microscale boehmite and gamma nanoleaves alumina. Mater Lett . 62, 1297-1301 (2008).

33. Kim, P., Kim, Y., Kim, H., Song, I.K. and Yi, J., Synthesis and characterization of mesoporous alumina for use as a catalyst support in the hydrodechlorination of 1,2dichloropropane:effect of preparation condition of mesoporous alumina. J. Mol Catal A: Chem. 219, 87-95 (2004).

34. Yada, M., Kitamura, H., Machida, M. and Kijima, T., Biomimetic surface patterns of layered aluminum oxide mesophases templated by mixed surfactant assemblies. Langmuir, 13, 5252-5257 (1997).

35. Cabrera, S., El-Haskouri, J. and Alamo, J., Surfactant-Assisted Synthesis of Mesoporous Alumina Showing Continuously Adjustable Pore Sizes. Adv Mater. 11, 379-381(1999).

36. Cejka, J., Zilkova, N., Rathousky, J. and Zukal, A., $\mathrm{N}_{2}$ adsorption study of organized mesoporous alumina. Phys Chem. Chem Phys. 3, 5076-5081(2001).

37. Alvine, K.J., Pontoni, D., Shpyrko, O.G., et al., Solvent mediated assembly of nanoparticles confined in mesoporous alumina. Phys. Rev. B. 73, 125412125421(2006).

38. Deng, W., Bodart, P., Pruski, M. and Shanks, B.H., Characterization of mesoporous alumina molecular sieves synthesized by nonionic templating. Microporous Mesoporous Mater. 52, 169-177(2002).

39. Naik B., Prasad V.S. and Ghosh N.N., Development of a simple aqueous solution based chemical method for synthesis of mesoporous $\gamma$-Alumina powders with disordered pore structure. J. Porous Mater. 17 (1), 115-121(2010).

40. Kresge, C.T., Leonowicz, M.E., Roth, W.J. and Vartuli, J.C., US5098684 (1992).

41. Beck, J.S., Vartuli, J.C. and Roth, W.J., A new family of mesoporous molecular sieves prepared with liquid crystal templates. J. Am. Chem. Soc. 114, 10834-10843 (1992).

42. Chen, C.Y., Li, H.X. and Davis, M.E., Studies on mesoporous materials: I. Synthesis and characterization of MCM-41. Microporous Mater., 2, 17- 26 (1993).

43. Steel, A., Carr, S.W. and Anderson, M.W., ${ }^{14}$ N NMR study of surfactant mesophases in the synthesis of mesoporous silicates. J. Chem. Soc. Chem. Commun.

Egypt. J. Chem. 59, No. 2 (2016) 
1571-1572 (1994).

44. Stucky, G.D., Monnier, A. and Schüth, F., et al. Molecular and atomic arrays in nano-and mesoporous materials synthesis. Mol. Cryst. Liq. Cryst. 240,187-200 (1994).

45. Firouzi, A., Atef, F., Oertli, A.G., Stucky, G.D. and Chmelka, B.F., Alkaline lyotropic silicate-surfactant liquid crystals. J. Am. Chem. Soc. 119, 3596-3610 (1997).

46. Huo, Q., Margolese, D.I. and Ciesla, U., et al. Organization of organic molecules with inorganic molecular species into nanocomposites biphase arrays. Chem. Mater. 6, 1176-1191 (1994).

47. Kim, J.M. and Stucky, G.D., Synthesis of highly ordered mesoporous silica materials using sodium silicate and amphiphilic block copolymers. Chem. Commun. 13, 1159-1160 (2000).

48. Yada, M., Machida, M. and Kijima, T., Synthesis and deorganization of of an aluminum based dodecyl sulfate mesophase with a hexagonal structure. Chem. Commun. 769-770 (1996).

49. Liu, Q., Wang, A., Wang, X., Gao, P., Wang, X. and Zhang, T., Synthesis, characterization and catalytic applications of mesoporous $\gamma$-alumina from boehmite sol. Microporous Mesoporous Mater. 111, 323-333(2008).

50. González-Peña, V., Díaz, I., Márquez-Alvarez, C., Sastre, E. and PérezPariente, J., Thermally stable mesoporous alumina synthesized with non-ionic surfactants in the presence of amines. Microporous Mesoporous Mater. 44-45 203210(2001).

51. Klotz, M., Ayral, A., Guizard C. and Cot, L., Synthesis conditions for hexagonal mesoporous silica layers. J. Mater. Chem. 10, 663-669 (2000).

52. Setoguchi, Y.M., Teraoka, Y., Moriguchi, I. and Kagawa, S., Rapid room temperature synthesis of hexagonal mesoporous silica using inorganic silicate sources and cationic surfactants under highly acidic condition. J. Porous Mater. 4, 129-134 (1997).

53. Mahmoud, S.A. and Gobara, H.M., Synthesis of nanostructured mesoporous ordered silica supported $\mathrm{Fe}_{2} \mathrm{O}_{3}$ nanoparticles for water purification "Processing of nanostructure materials and nanostructure films", Kathy $\mathrm{Lu}$, Chris $\mathrm{Li}$, Eugene Medvedoveski and Eugene A. Olevesky (Ed.) Ceramics Transaction, 233, The American Chemical Society, Wiley (2010).

(Received 28/12/2015; accepted $18 / 1 / 2016$ ) 


\section{ميكاتيزمات التحضير والتطبيقات المختلفة للمواد ذات المسام متوسطة الأحجام المبنية على أساس السيليكات والألومينا \\ هبة محمود جبارة

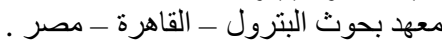

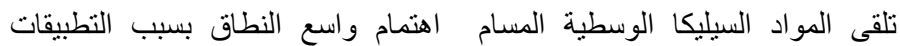

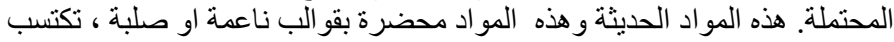

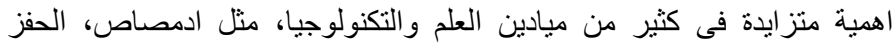

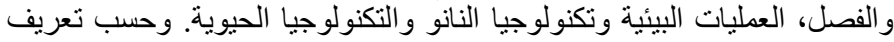

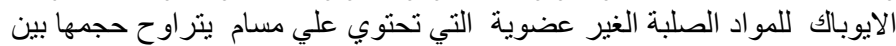

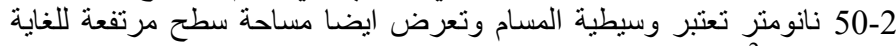

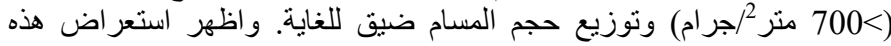

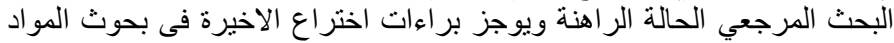

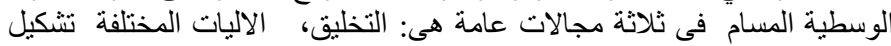

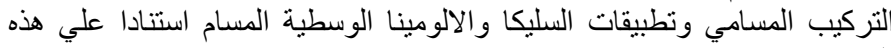

